\title{
Dynamic coupling between horizontal vessel motion and two-layer shallow-water sloshing
}

\author{
by H. Alemi Ardakani ${ }^{1}$, T.J. Bridges \& M.R. Turner \\ Department of Mathematics, University of Surrey, Guildford GU2 7XH, UK
}

\begin{abstract}
Numerical and analytical results are presented for fluid sloshing, of a two-layer inviscid, incompressible and immiscible fluid with thin layers and a rigid lid, coupled to a vessel which is free to undergo horizontal motion governed by a nonlinear spring. Exact analytical results are obtained for the linear problem, giving the natural frequencies and the resonance structure, particularly between the fluid and vessel. A numerical method for the linear and nonlinear equations is developed based on the high-resolution f-wave-propagation finite volume methods due to BAle, LeVeque, Mitran And Rossmanith (2002) (SIAM J. Sci. Comput. 24, 955-978), adapted to include the pressure gradient at the rigid-lid, and coupled to a Runge-Kutta solver for the vessel motion. The numerical simulations in the linear limit are compared with the exact analytical solutions. The coupled nonlinear numerical solutions with simulations near the internal $1: 1$ resonance are presented. Of particular interest is the partition of energy between the vessel and fluid motion.
\end{abstract}

\section{Introduction}

The effect of liquid sloshing on the dynamics and control of liquid transport, e.g. the terrestrial transport of liquids, oil and liquid natural gas in ships, and fuel in aviation and astrodynamics, has motivated a wide range of research. An indication of the breadth of research in this area can be found in the books of IBRAHIM [25] and FALTINSEN \& TIMOKHA [19] and references therein. The coupled dynamics between fluid sloshing and vessel motion brings in a new dimension and the potential for enhancing or diminishing the sloshing motion through vehicle dynamics. The coupled problem is of great practical interest in the transport of liquids along roads, maritime fluid transport, and industrial applications. Examples of where dynamic coupling is of interest is the sloshing of water on the the deck of fishing vessels [13], transport of liquid by robots [46, 47], motion planning for industrial control [23, 38, 20, 18], sloshing in automobile fuel tanks [48], and motion of water waves in a suspended container [16]. The book of MOISEYEV \& RUMYANTSEV [36] covers general aspects of the dynamic coupling between vessel motion and interior fluid motion.

The principal new directions in this paper are threefold: to consider an interior fluid with two layers of different density, include dynamic coupling with the vessel motion, and to include

\footnotetext{
${ }^{1}$ Corresponding author: email: h.alemiardakani@ surrey.ac.uk.
}

October 12,2015 
nonlinearity in both the vessel and fluid motion. Previous work on sloshing of two-layer fluids includes MACKEY \& COX [33], which derives a forced KdV equation and shows that forcing induces a form of chaos. Another example is the work of LA ROCCA ET AL. [39, 40] which includes both experiments and theory showing excellent agreement between a Lagrangian reduction to a large system of ODEs and the experimental results for a rigid lid container [39], and two layers with a free surface [40]. However, as far as we are aware there are no results in the literature which consider the dynamic coupling between a sloshing two-layer fluid and vessel motion. Here this coupled problem is considered with a shallow-water rigid-lid model for the two-layer fluid. There has been a vast amount of work on the two-layer model in open systems, and papers that have influenced the current theory are $[2,9,10,11,12,15,17,21,22$, 26, 27, 28, 29, 32, 34, 35, 37, 41, 42]. However the principal interest here is in closed systems. The introduction of two layers in an enclosed container with sloshing is motivated by a model for the Offshore Wave Energy Ltd (OWEL) wave energy converter [1].

For the dynamic coupling analysis a key precedent is the experiment of COOKER [16] on a horizontal rectangular wave tank which swings at the lower end of a bifilar pendulum such that the base of the tank remains horizontal. A linear analysis giving the natural frequencies of the coupled problem is given and compared with experiments in [16]. Cooker's model was extended in [5] by including a nonlinear fluid model coupled to the linear vessel motion. A Hamiltonian formulation, obtained by transforming to the Lagrangian particle-path (LPP) formulation of the fluid motion, is derived leading to a robust and fast numerical method with excellent energy conservation and partition properties. A key feature of [5] was the transformation to the LPP description, but a generalization of the LPP description to multi-layer fluids is difficult (see e.g. STEWART \& DELLAR [42]).

The theory for dynamic coupling with one layer was extended to the full $2 \mathrm{D}$ - not necessarily shallow - problem in [6] where a new $1: 1$ resonance structure was discovered. In the $1: 1$ resonance, one of the symmetric fluid sloshing mode frequencies is equal to the frequency of the anti-symmetric vessel-fluid coupled modes. In [24] experiments on the time-periodic liquid sloshing-induced sideways motion of containers are presented and the measurements are compared with the finite-depth potential theory developed from standard normal mode representations for rectangular boxes, upright cylinders, wedges and cones of $90^{\circ}$ apex angles, and cylindrical annuli. In [45] the nonlinear energy transfer between fluid sloshing and vessel motion is studied. The impact of sloshing on vehicle dynamics through the direct transfer of energy from fluid motion to vehicle motion is examined through resonance and nonlinearity. A nonlinear normal form is derived close to an internal $1: 1$ resonance, with the energy transfer manifested by a heteroclinic connection, which connects the purely symmetric sloshing modes to the purely antisymmetric sloshing modes. In [43] the dynamic coupling problem is studied for a suspended rectangular container from a single rigid pivoting rod and with interior partially filled with an inviscid fluid. A characteristic equation is derived for the natural frequencies of the coupled system and it is shown that the coupled pendulum sloshing dynamics exhibits unstable solutions if the length of the rigid pole is below some critical value.

An introduction to shallow-water multi-layer models is given in the book of BAINES [8]. They can be derived by integrating the Euler equations in the vertical coordinate direction. The model for the dynamic coupling to horizontal vessel motion is derived using a variational principle in $[3,5]$. The vessel with a rigid-lid is attached to a linear or nonlinear spring with the interior of the vessel housing a two-layer shallow-water sloshing fluid, as shown schematically in Figure 
1. The coupled equations are

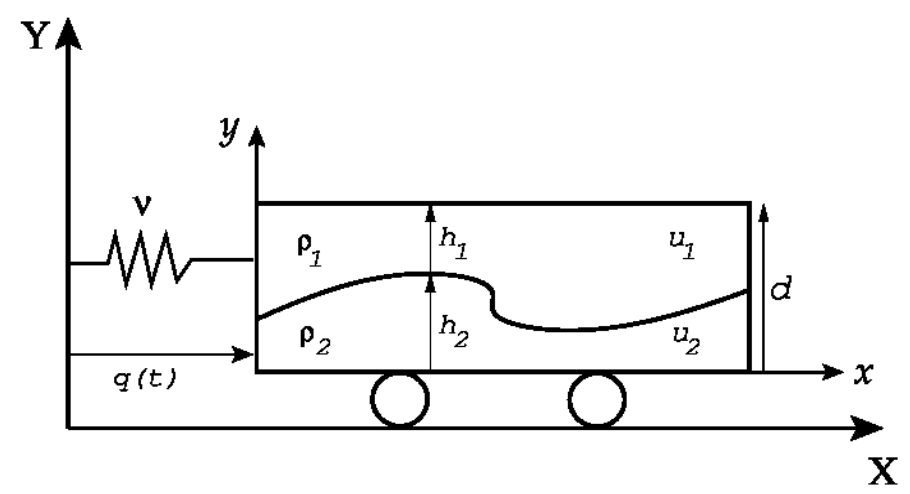

Figure 1: Schematic of a moving vessel with a rigid-lid constrained by a spring force and filled with two different fluids.

$$
\left.\begin{array}{l}
\left(\rho_{1} h_{1}\right)_{t}+\left(\rho_{1} h_{1} u_{1}\right)_{x}=0, \\
\left(\rho_{1} h_{1} u_{1}\right)_{t}+\left(\rho_{1} h_{1} u_{1}^{2}+\frac{1}{2} \rho_{1} g h_{1}^{2}\right)_{x}=-\rho_{1} g h_{1} h_{2 x}-h_{1} p_{x}-\rho_{1} h_{1} \ddot{q}, \\
\left(\rho_{2} h_{2}\right)_{t}+\left(\rho_{2} h_{2} u_{2}\right)_{x}=0, \\
\left(\rho_{2} h_{2} u_{2}\right)_{t}+\left(\rho_{2} h_{2} u_{2}^{2}+\frac{1}{2} \rho_{2} g h_{2}^{2}\right)_{x}=-\rho_{1} g h_{2} h_{1 x}-h_{2} p_{x}-\rho_{2} h_{2} \ddot{q}, \\
h_{1}(x, t)+h_{2}(x, t)=d, \\
\frac{d}{d t}\left(\int_{0}^{L}\left(\rho_{1} h_{1} u_{1}+\rho_{2} h_{2} u_{2}\right) d x+\left(m_{f}^{1}+m_{f}^{2}+m_{v}\right) \dot{q}\right)+\nu_{1} q-\nu_{2} q^{3}=0,
\end{array}\right\}
$$

in $0 \leq x \leq L$, with boundary conditions

$$
u_{j}(0, t)=u_{j}(L, t)=0, \quad \text { for } j=1,2 .
$$

In the equations, $h_{1}, u_{1}$ and $\rho_{1}$ denote the depth, velocity and density of the upper layer, and $h_{2}, u_{2}$ and $\rho_{2}$ correspond to the lower layer. The pressure at the rigid lid is denoted by $p$ and $g$ is the gravitational constant. There are two frames of reference: the spatial (inertial) frame has coordinates $\mathbf{X}=(X, Y)$ and the body frame has coordinates $\mathbf{x}=(x, y)$. The whole system has a uniform translation, denoted $q(t)$, in the $x$-direction; hence

$$
X=x+q \quad \text { and } \quad Y=y .
$$

The fifth equation in (1.1) is the rigid-lid constraint with $d$ a given positive constant. The first and third equations in (1.1) are conservation of mass, and the second and fourth equations are the conservation of momentum for each layer. The result of the vertical integration and hydrostatic assumption is a system of partial differential equations similar to two sets of one-layer shallowwater equations with the addition of a coupling term between the layers. This coupling term is due to the integration of the hydrostatic pressure and does not represent momentum transfer due to drag force between the layers [35]. The last equation in (1.1) is the governing nonlinear equation for the motion of the vessel where $\nu_{1}>0$ and $\nu_{2}$ are constants. If $\nu_{2}<0$ the spring 
is called hard and if $\nu_{2}>0$ it is called soft. Also $m_{f}^{1}, m_{f}^{2}$ and $m_{v}$ are, respectively, the mass of the upper layer fluid, the mass of the lower layer fluid and the mass of the vessel.

Another form of the coupled two-layer shallow water system (1.1) is obtained by replacing the fourth equation of (1.1) by

$$
\left(\rho_{2} h_{2} u_{2}\right)_{t}+\left(\rho_{2} h_{2} u_{2}^{2}+\frac{1}{2} \rho_{2} g h_{2}^{2}+\rho_{1} g h_{1} h_{2}\right)_{x}=\rho_{1} g h_{1} h_{2 x}-h_{2} p_{x}-\rho_{2} h_{2} \ddot{q},
$$

where an extra term appears in the flux of the lower layer momentum equation and the nonconservative coupling terms in each layer become symmetric $[2,35]$. This equation is equivalent to the fourth equation of (1.1), but has advantages for the numerics. The symmetry in the nonconservative products has the benefit that the transfer of momentum due to these coupling terms moves directly between the layers which is advantageous in numerical integration [35].

A class of high resolution wave-propagation finite volume methods are developed in [30] for multidimensional hyperbolic systems. These methods are based on solving Riemann problems for waves that define both first order updates to cell averages and also second order corrections which can be modified by limiter functions to obtain high resolution numerical solutions. The wave-propagation algorithms are modified in [9] for conservation laws and balance laws with spatially varying flux functions and called f-wave-propagation methods. The main novel feature of the modified algorithms is to solve the Riemann problems by decomposition of the jump in the flux functions into waves propagating out from each grid cell interface instead of decomposition of the jump in cell averages. In [21, 22] a class of augmented approximate Riemann solvers is developed for the single layer shallow water equations in the presence of a variable bottom surface using the f-wave-propagation algorithm. The solver is based on a decomposition of an augmented solution vector including the depth, momentum, momentum flux and the bottom surface. This solver is well-balanced, maintains depth non-negativity and extends to Riemann problems with an initial dry state. In [34, 35] the f-wave-propagation finite volume method is used to develop solvers for the multilayer shallow water equations in one and two dimensions. The proposed approximate Riemann solvers also handle dry states in the system where the bottom layer depth becomes zero. In [28] the two-layer shallow water system is studied using the f-wave methods. It is discussed that the two-layer system is conditionally hyperbolic because of the coupling terms between the layers. These terms may cause the eigenvalues to become imaginary. In [27] the f-wave method is used to solve the wave propagation problems generated by submarine landslides. Also several types of the Boussinesq equations are reviewed and implemented with a hybrid of high-resolution finite volume and finite difference methods. In [7] the high-resolution f-wave-propagation finite volume methods of [9] are adapted to the two-layer inviscid, incompressible and immiscible shallow-water equations in a moving vessel with a rigid-lid. The main novel features of this paper are the inclusion of the exact expression for the pressure gradient at the rigid-lid into the numerical calculations, the inclusion of time-dependent source terms, and the coupling to vessel motion.

An outline of the paper is as follows. In $\S 2$ an exact analysis of the linear problem is given resulting in the characteristic equation. The characteristic equation has a product structure as in [6] with a $1: 1$ resonance along lines in parameter space. In $\S 3$ the governing equations for the fluid are analysed in preparation for the numerics. In $\$ 4$ the details of the numerical scheme for the fluid are presented. The scheme is based on the algorithm of BALE ET AL. [9] suitably modified to take into account the rigid lid and the coupling to the vessel motion. The vessel motion is governed by the scalar $q(t)$ and when the fluid motion is given, $q(t)$ satisfies 
an ordinary differential equation. In $\S 5$ an algorithm for integrating the $q$ - equation is given. It is based on a Runge-Kutta algorithm and is implemented using MATLAB's ODE45. Results of simulations of the coupled problem are reported in $\$ 6.1$ and $\$ 6.2$. The key feature of the linear simulations is comparison with the exact solution, and the key feature of the nonlinear simulations is energy partition between the vessel and fluid. In $§ 7$ a summary and discussion of how the results apply to ocean wave energy converters is given.

\section{The linear coupled problem and natural frequencies}

An analysis of the linear problem will be useful for determining how the natural frequencies of the two-layer shallow-water sloshing inside a container with a rigid-lid are modified by the coupling. See [7] for the natural frequencies of the two layer shallow water sloshing problem without the vessel equation. The exact linear solutions will be used to validate the numerical solutions.

Consider the linearisation of system (1.1) about the trivial state

$$
\begin{aligned}
& h_{1}(x, t)=h_{1}^{0}+\widetilde{h}_{1}(x, t), \quad h_{2}(x, t)=h_{2}^{0}+\widetilde{h}_{2}(x, t), \quad u_{1}(x, t)=\widetilde{u}_{1}(x, t), \\
& u_{2}(x, t)=\widetilde{u}_{2}(x, t), \quad p(x, t)=\widetilde{p}(x, t), \quad q(t)=\widetilde{q}(t) .
\end{aligned}
$$

The linearised equations are

$$
\left.\begin{array}{l}
\left(\rho_{1} \widetilde{h}_{1}\right)_{t}+\left(\rho_{1} h_{1}^{0} \widetilde{u}_{1}\right)_{x}=0, \\
\left(\rho_{1} h_{1}^{0} \widetilde{u}_{1}\right)_{t}+\left(\rho_{1} g h_{1}^{0} \widetilde{h}_{1}\right)_{x}=-\rho_{1} g h_{1}^{0}\left(\widetilde{h}_{2}\right)_{x}-h_{1}^{0} \widetilde{p}_{x}-\rho_{1} h_{1}^{0} \ddot{\widetilde{q}}, \\
\left(\rho_{2} \widetilde{h}_{2}\right)_{t}+\left(\rho_{2} h_{2}^{0} \widetilde{u}_{2}\right)_{x}=0, \\
\left(\rho_{2} h_{2}^{0} \widetilde{u}_{2}\right)_{t}+\left(\rho_{2} g h_{2}^{0} \widetilde{h}_{2}\right)_{x}=-\rho_{1} g h_{2}^{0}\left(\widetilde{h}_{1}\right)_{x}-h_{2}^{0} \widetilde{p}_{x}-\rho_{2} h_{2}^{0} \ddot{\widetilde{q}}, \\
\widetilde{h}_{1}(x, t)+\widetilde{h}_{2}(x, t)=0, \\
h_{1}^{0}+h_{2}^{0}=d, \\
\frac{d}{d t}\left(\int_{0}^{L}\left(\rho_{1} h_{1}^{0} \widetilde{u}_{1}+\rho_{2} h_{2}^{0} \widetilde{u}_{2}\right) d x+\left(m_{f}^{1}+m_{f}^{2}+m_{v}\right) \dot{\widetilde{q}}\right)+\nu \widetilde{q}=0,
\end{array}\right\}
$$

where $\nu=\nu_{1}, m_{f}^{1}=\rho_{1} h_{1}^{0} L$, and $m_{f}^{2}=\rho_{2} h_{2}^{0} L$. This system can be written as

$$
\widetilde{\mathrm{q}}_{t}+A(\widetilde{\mathrm{q}}) \widetilde{\mathrm{q}}_{x}=\widehat{\Psi}(\widetilde{\mathrm{q}}, t)
$$

where

$$
\widetilde{\mathbf{q}}=\left[\begin{array}{c}
\rho_{1} \widetilde{h}_{1} \\
\rho_{1} h_{1}^{0} \widetilde{u}_{1} \\
\rho_{2} \widetilde{h}_{2} \\
\rho_{2} h_{2}^{0} \widetilde{u}_{2}
\end{array}\right], \quad A(\widetilde{\mathbf{q}})=\left[\begin{array}{cccc}
0 & 1 & 0 & 0 \\
g h_{1}^{0} & 0 & r g h_{1}^{0} & 0 \\
0 & 0 & 0 & 1 \\
g h_{2}^{0} & 0 & g h_{2}^{0} & 0
\end{array}\right], \quad \widehat{\Psi}(\widetilde{\mathbf{q}}, t)=\left[\begin{array}{c}
0 \\
-h_{1}^{0} \widetilde{p}_{x}-\rho_{1} h_{1}^{0} \ddot{\widetilde{q}} \\
0 \\
-h_{2}^{0} \widetilde{p}_{x}-\rho_{2} h_{2}^{0} \ddot{\widetilde{q}}
\end{array}\right],
$$


$r=\rho_{1} / \rho_{2}$ and with the eigenstructure given in (3.12) and (3.13). Differentiating the rigid-lid constraint equation $\widetilde{h}_{1}+\widetilde{h}_{2}=0$ with respect to time and using the mass equations in (2.1) gives

$$
\partial_{x}\left(h_{1}^{0} \widetilde{u}_{1}+h_{2}^{0} \widetilde{u}_{2}\right)=0,
$$

and so $h_{1}^{0} \widetilde{u}_{1}+h_{2}^{0} \widetilde{u}_{2}$ is independent of $x$. With the boundary conditions

$$
\widetilde{u}_{1}(0, t)=\widetilde{u}_{1}(L, t)=0 \quad \text { and } \quad \widetilde{u}_{2}(0, t)=\widetilde{u}_{2}(L, t)=0,
$$

it follows that $h_{1}^{0} \widetilde{u}_{1}+h_{2}^{0} \widetilde{u}_{2}=0$. Now differentiate this function with respect to time and use the momentum equations in (2.1) to get an equation for the pressure gradient at the rigid-lid

$$
\frac{\partial \widetilde{p}}{\partial x}=\frac{\rho_{1} \rho_{2}}{\rho_{2} h_{1}^{0}+\rho_{1} h_{2}^{0}}\left(-g h_{2}^{0}\left(\frac{\partial \widetilde{h}_{2}}{\partial x}+r \frac{\partial \widetilde{h}_{1}}{\partial x}\right)-d \ddot{\widetilde{q}}\right) .
$$

Introduce harmonic solutions of the following form

$$
\begin{aligned}
\widetilde{h}_{1}(x, t) & =H_{1}(x) \cos \omega t, \quad \widetilde{h}_{2}(x, t)=H_{2}(x) \cos \omega t, \\
\widetilde{u}_{1}(x, t) & =A_{1}(x) \sin \omega t, \quad \widetilde{u}_{2}(x, t)=A_{2}(x) \sin \omega t, \\
\widetilde{p}(x, t) & =\widehat{p}(x) \cos \omega t, \quad \widetilde{q}=\widehat{q} \cos \omega t .
\end{aligned}
$$

Substitution into the linear equations (2.1) then gives

$$
\left.\begin{array}{l}
H_{1}(x)+H_{2}(x)=0, \quad h_{1}^{0}+h_{2}^{0}=d, \\
-H_{1} \omega+h_{1}^{0} \frac{d A_{1}}{d x}=0, \quad-H_{2} \omega+h_{2}^{0} \frac{d A_{2}}{d x}=0, \\
A_{1} \omega-\widehat{q} \omega^{2}+\frac{1}{\rho_{1}} \frac{d \widehat{p}}{d x}=0, \\
A_{2} \omega-\widehat{q} \omega^{2}+g \frac{d H_{2}}{d x}+r g \frac{d H_{1}}{d x}+\frac{1}{\rho_{2}} \frac{d \widehat{p}}{d x}=0, \\
\int_{0}^{L} \rho_{1} h_{1}^{0} \omega A_{1} d x+\int_{0}^{L} \rho_{2} h_{2}^{0} \omega A_{2} d x-\left(m_{f}^{1}+m_{f}^{2}+m_{v}\right) \widehat{q} \omega^{2}+\nu \widehat{q}=0 .
\end{array}\right\}
$$

The boundary conditions (2.4) require $A_{1}(0)=A_{2}(0)=0$ and so the constraint (2.3) gives $h_{1}^{0} A_{1}+h_{2}^{0} A_{2}=0$. Hence

$$
A_{2}(x)=-\frac{h_{1}^{0}}{h_{2}^{0}} A_{1}(x) \quad \text { and } \quad H_{2}(x)=-H_{1}(x)=-\frac{h_{1}^{0}}{\omega} \frac{d A_{1}}{d x} .
$$

Next, eliminate $\widehat{p}_{x}$ from (2.7) to get

$$
\omega^{2} \rho_{1} \widehat{q}-\omega \rho_{1} A_{1}+\omega \rho_{2} A_{2}-\omega^{2} \rho_{2} \widehat{q}+\rho_{2} g \frac{d H_{2}}{d x}+\rho_{1} g \frac{d H_{1}}{d x}=0,
$$

or after substituting for $A_{2}$ and noting that $\frac{d H_{2}}{d x}=-\frac{d H_{1}}{d x}$,

$$
-\left(\rho_{1}+\rho_{2} \frac{h_{1}^{0}}{h_{2}^{0}}\right) \omega A_{1}+\left(\rho_{1}-\rho_{2}\right) \omega^{2} \widehat{q}+\left(\rho_{1}-\rho_{2}\right) g \frac{d H_{1}}{d x}=0 .
$$


Now substituting for $\frac{d H_{1}}{d x}$, this latter equation modifies to

$$
\frac{d^{2} A_{1}}{d x^{2}}+\alpha^{2} A_{1}+\frac{\omega^{3}}{g h_{1}^{0}} \widehat{q}=0
$$

where

$$
\alpha^{2}=\frac{\left(\rho_{1} h_{2}^{0}+\rho_{2} h_{1}^{0}\right)}{\left(\rho_{2}-\rho_{1}\right) g h_{1}^{0} h_{2}^{0}} \omega^{2}
$$

with the boundary conditions $A_{1}(0)=A_{1}(L)=0$. Solving for $A_{1}$ then gives

$$
A_{1}(x)=F \cos \alpha x+G \sin \alpha x-\frac{\omega^{3} \widehat{q}}{g h_{1}^{0} \alpha^{2}} .
$$

The boundary condition at $x=0$ gives

$$
A_{1}(x)=\frac{\omega^{3} \widehat{q}}{g h_{1}^{0} \alpha^{2}} \cos \alpha x+G \sin \alpha x-\frac{\omega^{3} \widehat{q}}{g h_{1}^{0} \alpha^{2}},
$$

and the boundary condition at $x=L$ gives

$$
-\frac{\omega^{3} \widehat{q}}{g h_{1}^{0} \alpha^{2}} \sin ^{2}\left(\frac{1}{2} \alpha L\right)+G \sin \left(\frac{1}{2} \alpha L\right) \cos \left(\frac{1}{2} \alpha L\right)=0 .
$$

To evaluate the vessel equation in (2.7) integrate $A_{1}(x)$,

$$
\begin{aligned}
\int_{0}^{L} A_{1}(x) d x & =\int_{0}^{L}\left(\frac{\omega^{3} \widehat{q}}{g h_{1}^{0} \alpha^{2}} \cos \alpha x+G \sin \alpha x-\frac{\omega^{3} \widehat{q}}{g h_{1}^{0} \alpha^{2}}\right) d x \\
& =\frac{\omega^{3} \widehat{q}}{g h_{1}^{0} \alpha^{3}} \sin (\alpha L)-\frac{\omega^{3} \widehat{q} L}{g h_{1}^{0} \alpha^{2}}+\frac{G}{\alpha}(1-\cos (\alpha L)),
\end{aligned}
$$

and so

$$
\int_{0}^{L} A_{2}(x) d x=-\frac{h_{1}^{0}}{h_{2}^{0}}\left(\frac{\omega^{3} \widehat{q}}{g h_{1}^{0} \alpha^{3}} \sin (\alpha L)-\frac{\omega^{3} \widehat{q} L}{g h_{1}^{0} \alpha^{2}}+\frac{G}{\alpha}(1-\cos (\alpha L))\right) .
$$

Hence the vessel equation modifies to

$$
\begin{gathered}
\left(2\left(\rho_{1}-\rho_{2}\right) \frac{\omega^{4}}{g \alpha^{3}} \sin \left(\frac{1}{2} \alpha L\right) \cos \left(\frac{1}{2} \alpha L\right)+\left(\rho_{2}-\rho_{1}\right) \frac{\omega^{4} L}{g \alpha^{2}}-\left(m_{f}^{1}+m_{f}^{2}+m_{v}\right) \omega^{2}+\nu\right) \widehat{q} \\
+2\left(\rho_{1}-\rho_{2}\right) \frac{h_{1}^{0} \omega}{\alpha} \sin ^{2}\left(\frac{1}{2} \alpha L\right) G=0 .
\end{gathered}
$$

Equations (2.10) and (2.11) are two homogeneous equations for two unknowns. Combining them into one matrix equation gives

$$
\left[\begin{array}{cc}
\frac{-2 \omega^{3}}{g h_{1}^{0} \alpha^{2}} \sin ^{2}\left(\frac{1}{2} \alpha L\right) & 2 \sin \left(\frac{1}{2} \alpha L\right) \cos \left(\frac{1}{2} \alpha L\right) \\
\mathrm{C} & 2\left(\rho_{1}-\rho_{2}\right) \frac{h_{1}^{0} \omega}{\alpha} \sin ^{2}\left(\frac{1}{2} \alpha L\right)
\end{array}\right]\left[\begin{array}{l}
\widehat{q} \\
G
\end{array}\right]=\left[\begin{array}{l}
0 \\
0
\end{array}\right],
$$


where

$$
\mathrm{C}=2\left(\rho_{1}-\rho_{2}\right) \frac{\omega^{4}}{g \alpha^{3}} \sin \left(\frac{1}{2} \alpha L\right) \cos \left(\frac{1}{2} \alpha L\right)+\left(\rho_{2}-\rho_{1}\right) \frac{\omega^{4} L}{g \alpha^{2}}-\left(m_{f}^{1}+m_{f}^{2}+m_{v}\right) \omega^{2}+\nu .
$$

For non-trivial solutions the determinant of the coefficient matrix must vanish, resulting

$$
\frac{-4 \omega^{4}}{g \alpha^{3}}\left(\rho_{1}-\rho_{2}\right) \sin ^{4}\left(\frac{1}{2} \alpha L\right)-2 \sin \left(\frac{1}{2} \alpha L\right) \cos \left(\frac{1}{2} \alpha L\right) \mathrm{C}=0 .
$$

Let $s=\frac{1}{2} \alpha L$ then

$$
\begin{aligned}
\Delta(s)= & -\frac{4 \omega^{4}}{g \alpha^{3}}\left(\rho_{1}-\rho_{2}\right) \sin ^{2}(s)+\frac{2 \omega^{4} L}{g \alpha^{2}}\left(\rho_{1}-\rho_{2}\right) \sin (s) \cos (s) \\
& +2\left(m_{f}^{1}+m_{f}^{2}+m_{v}\right) \omega^{2} \sin (s) \cos (s)-2 \nu \sin (s) \cos (s)=0 .
\end{aligned}
$$

However, if $\cos (s)=0$ then $\Delta(s) \neq 0$. Hence $\cos (s)$ is never zero, and it can be divided out, and so $\Delta(s)$ reduces to

$$
\begin{aligned}
\Delta(s)= & -\frac{4 \omega^{4}}{g \alpha^{3}}\left(\rho_{1}-\rho_{2}\right) \sin (s) \tan (s)+\frac{2 \omega^{4} L}{g \alpha^{2}}\left(\rho_{1}-\rho_{2}\right) \sin (s) \\
& +2\left(m_{f}^{1}+m_{f}^{2}+m_{v}\right) \omega^{2} \sin (s)-2 \nu \sin (s)=0,
\end{aligned}
$$

or after substituting for $\omega$ and $\alpha$ in terms of $s$,

$$
\Delta(s)=\mathrm{P}(s) \mathrm{D}(s),
$$

where

$$
\begin{aligned}
\mathrm{P}(s)= & \sin (s) \\
\mathrm{D}(s)= & \frac{4}{g L}\left(\rho_{1}-\rho_{2}\right)\left(\frac{\left(\rho_{2}-\rho_{1}\right) g h_{1}^{0} h_{2}^{0}}{\rho_{1} h_{2}^{0}+\rho_{2} h_{1}^{0}}\right)^{2}\left(s^{2}-s \tan (s)\right) \\
& +\frac{4}{L^{2}}\left(m_{f}^{1}+m_{f}^{2}+m_{v}\right) \frac{\left(\rho_{2}-\rho_{1}\right) g h_{1}^{0} h_{2}^{0}}{\rho_{1} h_{2}^{0}+\rho_{2} h_{1}^{0}} s^{2}-\nu
\end{aligned}
$$

Also $\mathrm{D}(s)$ can be converted to the following form,

$$
\mathrm{D}(s)=\frac{\mathrm{G}}{s}-\mathrm{R} s-\tan (s),
$$

with

$$
\mathrm{G}=\frac{g L \nu}{4\left(\rho_{2}-\rho_{1}\right)^{3}\left(\frac{g h_{1}^{0} h_{2}^{0}}{\rho_{1} h_{2}^{0}+\rho_{2} h_{1}^{0}}\right)^{2}} \quad \text { and } \quad \mathrm{R}=-1+\frac{\left(m_{f}^{1}+m_{f}^{2}+m_{v}\right)\left(\rho_{1} h_{2}^{0}+\rho_{2} h_{1}^{0}\right)}{L\left(\rho_{2}-\rho_{1}\right)^{2} h_{1}^{0} h_{2}^{0}}
$$

and by factoring out an $s$ term which we assume is non-zero. The function $\mathrm{D}(s)$ is similar to the characteristic function first derived in [16] for the dynamic coupling problem with one-layer shallow-water equations with a free surface, but with different $G$ and $R$ here. With two factors in the characteristic function (2.13) there are three principal classes of solutions: 
1. $\mathrm{D}(s)=0$ but $\frac{d}{d s} \mathrm{D}(s) \neq 0$ and $\mathrm{P}(s) \neq 0$ : anti-symmetric fluid mode coupled to vessel motion.

2. $\mathrm{P}(s)=0$ but $\frac{d}{d s} \mathrm{P}(s) \neq 0$ and $\mathrm{D}(s) \neq 0$ : symmetric fluid mode decoupled from vessel motion.

3. $\mathrm{D}(s)=0$ and $\mathrm{P}(s)=0$ but $\frac{d}{d s} \mathrm{D}(s) \neq 0$ and $\frac{d}{d s} \mathrm{P}(s) \neq 0$ : internal $1: 1$ resonance between a symmetric and anti-symmetric fluid mode which is coupled to the vessel motion.

\subsection{Solution class 1: $\mathrm{D}(s)=0, \mathrm{P}(s) \neq 0$}

The first class of modes are associated with roots of $\mathrm{D}(s)$ with the conditions

$$
\mathrm{D}(s)=0 \quad \text { but } \quad \frac{d}{d s} \mathrm{D}(s) \neq 0 \quad \text { and } \quad \mathrm{P}(s) \neq 0 .
$$

These are the modes which fully couple the vessel and fluid motion. An explicit solution for $\mathrm{D}(s)=0$ has not been found, but the qualitative position of the roots can be established by plotting G/s - Rs and $\tan (s)$ (see Figure 2 in [16]). The second condition in (2.15) is satisfied since

$$
\frac{d}{d s} \mathrm{D}(s)=-\frac{\mathrm{G}}{s^{2}}-\mathrm{R}-1-\tan ^{2}(s),
$$

which is not zero for any $s>0$. The third condition in (2.15) is satisfied as long as

$$
\mathrm{G} \neq m^{2} \pi^{2} \mathrm{R} \quad \text { for any } \quad m \in \mathbb{N} .
$$

The mode shapes are determined as follows. In this case $G$ and $\widehat{q}$ in (2.10) are related by

$$
G=\frac{\omega^{3} \tan (s)}{g h_{1}^{0} \alpha^{2}} \widehat{q}
$$

and hence

$$
A_{1}(x)=\frac{\omega^{3}}{g h_{1}^{0} \alpha^{2}}[\cos (\alpha x)+\tan (s) \sin (\alpha x)-1] \widehat{q},
$$

with the interface

$$
H_{1}(x)=-\frac{\omega^{2}}{g \alpha}[\sin (\alpha x)-\tan (s) \cos (\alpha x)] \widehat{q},
$$

with the value of $\widehat{q}$ arbitrary, determined by the initial data, and the value of $s$ (and $\alpha=2 s / L$ ) determined via one of the roots of $\mathrm{D}(s)=0$. To see that the interface is anti-symmetric about the centreline, rewrite $H_{1}(x)$ in terms of $x-\frac{1}{2} L$,

$$
\sin (\alpha x)-\tan (s) \cos (\alpha x)=\frac{1}{\cos (s)} \sin \left(\alpha\left(x-\frac{1}{2} L\right)\right)
$$

and hence

$$
H_{1}(x)=-\frac{\omega^{2} \widehat{q}}{g \alpha} \frac{1}{\cos (s)} \sin \left(\alpha\left(x-\frac{1}{2} L\right)\right)
$$


and so $H_{1}(0)=-H_{1}(L)$. The equation $\mathrm{D}(s)=0$ has a countable number of positive solutions, $s_{j}$ with $j=1,2, \ldots$. Given a root $s_{j}$ satisfying $\mathrm{D}\left(s_{j}\right)=0$, the natural frequency of the coupled system is given by

$$
\omega_{j}^{\text {coupled }}=\frac{2}{L} \sqrt{\frac{\left(\rho_{2}-\rho_{1}\right) g h_{1}^{0} h_{2}^{0}}{\rho_{2} h_{1}^{0}+\rho_{1} h_{2}^{0}}} s_{j} .
$$

For comparison, the natural frequencies of the uncoupled problem are recorded here. The natural frequencies of the two-layer fluid when the vessel is fixed (the sloshing frequencies) are [7]

$$
\omega_{j}^{f}=\frac{2}{L} \sqrt{\frac{\left(\rho_{2}-\rho_{1}\right) g h_{1}^{0} h_{2}^{0}}{\rho_{2} h_{1}^{0}+\rho_{1} h_{2}^{0}}} \frac{j \pi}{2}, \quad j=1,2, \ldots .
$$

The natural frequency of the dry vessel plus two fluids with the fluids treated as rigid-body is

$$
\omega_{f v}=\sqrt{\frac{\nu}{m_{v}+m_{f}^{1}+m_{f}^{2}}}=\frac{2}{L} \sqrt{\frac{\left(\rho_{2}-\rho_{1}\right) g h_{1}^{0} h_{2}^{0}}{\rho_{2} h_{1}^{0}+\rho_{1} h_{2}^{0}}} \sqrt{\frac{\mathrm{G}}{1+\mathrm{R}}} .
$$

It can be shown that $\omega_{1}^{\text {coupled }}$ is strictly less than all other natural frequencies:

$$
\omega_{1}^{\text {coupled }}<\omega_{1}^{f}, \quad \omega_{1}^{\text {coupled }}<\omega_{f v} .
$$

The first inequality follows from the fact that for strictly positive $G$ and $R$ the fundamental root lies in the interval $0<s_{1}<\frac{1}{2} \pi$ [16]. Furthermore, $\omega_{1}^{\text {coupled }} \rightarrow \omega_{1}^{f}$ only in the limit $G \rightarrow \infty$. The second inequality in (2.20) is verified as follows. Use the inequality $\tan \left(s_{1}\right)>s_{1}$ (since $\left.2 s_{1}<\pi\right)$ and then

$$
\frac{\mathrm{G}}{s_{1}}-\mathrm{R} s_{1}=\tan \left(s_{1}\right)>s_{1} \Rightarrow s_{1}<\sqrt{\frac{\mathrm{G}}{1+\mathrm{R}}} \Rightarrow \omega_{1}^{\text {coupled }}<\omega_{f v} .
$$

So the important conclusion is that when the vessel oscillates with a wave in its fundamental mode (wave mode) the frequency is less than the two-fluid frequency with vessel held fixed, and the frequency of the dry vessel plus the two fluids treated as rigid-body.

\subsection{Solution class 2: $\mathrm{P}(s)=0, \mathrm{D}(s) \neq 0$}

The second class of modes are associated with roots of $\mathrm{P}(s)$ with the conditions

$$
\mathrm{P}(s)=0 \quad \text { but } \quad \frac{d}{d s} \mathrm{P}(s) \neq 0 \quad \text { and } \quad \mathrm{D}(s) \neq 0 .
$$

Setting $\mathrm{P}(s)=0$ gives $\sin (s)=0$ and so $s=m \pi$ for some $m \in \mathbb{N}$. The second condition in (2.22) is satisfied since $\frac{d}{d s} \mathrm{P}(s)=\cos (s)$ which is non-zero when $s=m \pi$. The third condition in (2.22) is satisfied as long as (2.16) is satisfied.

With $\mathrm{D}(s) \neq 0$ it follows from (2.11) that $\widehat{q}=0$ and so the vessel is stationary. The $m-$ dependent velocity solution is

$$
A_{1}(x)=G_{m} \sin \left(2 m \pi \frac{x}{L}\right)
$$


with $G_{m}$ arbitrary, determined by the initial conditions. The $m$-dependent interface mode shape is

$$
H_{1}(x)=\frac{h_{1}^{0}}{\omega} \frac{2 m \pi}{L} G_{m} \cos \left(2 m \pi \frac{x}{L}\right) .
$$

The symmetry of this mode follows from the fact that $H_{1}(0)=H_{1}(L)$.

\subsection{Solution class 3: $\mathrm{D}(s)=\mathrm{P}(s)=0$}

The conditions for this class of solutions are

$$
\mathrm{P}(s)=0 \quad \text { and } \quad \mathrm{D}(s)=0 \quad \text { but } \quad \frac{d}{d s} \mathrm{P}(s) \neq 0 \quad \text { and } \quad \frac{d}{d s} \mathrm{D}(s) \neq 0 .
$$

The conditions (2.23) are equivalent to

$$
\Delta(s)=\frac{d}{d s} \Delta(s)=0
$$

which is the usual necessary condition for a $1: 1$ resonance. It is also sufficient since the eigenfunction associated with $\mathrm{P}(s)=0$ is linearly independent from the eigenfunction associated with $\mathrm{D}(s)=0$.

The requirement $\mathrm{P}(s)=0$ gives $s=m \pi$ for some $m \in \mathbb{N}$. Substituting into the second factor

$$
\mathrm{D}(m \pi)=\frac{\mathrm{G}}{m \pi}-\mathrm{R} m \pi-\tan (m \pi)=\frac{\mathrm{G}}{m \pi}-\mathrm{R} m \pi,
$$

and hence the condition for the internal $1: 1$ resonance is a condition on the parameters $G$ and $\mathrm{R}$,

$$
\mathrm{G}=m^{2} \pi^{2} \mathrm{R}, \quad \text { for some } \quad m \in \mathbb{N} .
$$

The third and fourth conditions in (2.23) are satisfied as noted in $\S 2.1$ and $\S 2.2$. In terms of the physical parameters the value of $\nu$ for the resonance condition is

$$
\nu_{1: 1}=\frac{4 m^{2} \pi^{2}}{g L}\left(-1+\frac{\left(m_{f}^{1}+m_{f}^{2}+m_{v}\right)\left(\rho_{1} h_{2}^{0}+\rho_{2} h_{1}^{0}\right)}{L\left(\rho_{2}-\rho_{1}\right)^{2} h_{1}^{0} h_{2}^{0}}\right)\left(\rho_{2}-\rho_{1}\right)^{3}\left(\frac{g h_{1}^{0} h_{2}^{0}}{\rho_{1} h_{2}^{0}+\rho_{2} h_{1}^{0}}\right)^{2} .
$$

With all the parameters on the right hand side, there is a much wider range of possible physical values of $\nu$ that can give rise to the $1: 1$ resonance.

At resonance the vessel natural frequency equals one of the symmetric modes of the fluid oscillation. The symmetric fluid modes exert no horizontal force on the vessel. However, at resonance, these symmetric modes can mix with the vessel motion. For each $m \in \mathbb{N}$, there is a 
continuum of such solutions in the linear problem, with eigenfunctions

$$
\begin{aligned}
& \int \widetilde{u}_{1}^{m}(x, t)=2 \sin \left(\frac{1}{2} m \kappa x\right)\left(-\frac{m \kappa}{g h_{1}^{0}}\left(\frac{\left(\rho_{2}-\rho_{1}\right) g h_{1}^{0} h_{2}^{0}}{\rho_{1} h_{2}^{0}+\rho_{2} h_{1}^{0}}\right)^{\frac{3}{2}} \sin \left(\frac{1}{2} m \kappa x\right) \widehat{q}_{m}\right. \\
& \left.+G_{m} \cos \left(\frac{1}{2} m \kappa x\right)\right) \sin \left(\omega_{m} t\right), \\
& \left\{\widetilde{h}_{1}^{m}(x, t)=\left(-m \kappa \frac{\left(\rho_{2}-\rho_{1}\right) h_{1}^{0} h_{2}^{0}}{\rho_{1} h_{2}^{0}+\rho_{2} h_{1}^{0}} \sin (m \kappa x) \widehat{q}_{m}+\frac{G_{m} h_{1}^{0}}{\left(\frac{\left(\rho_{2}-\rho_{1}\right) g h_{1}^{0} h_{2}^{0}}{\rho_{1} h_{2}^{0}+\rho_{2} h_{1}^{0}}\right)^{\frac{1}{2}}} \cos (m \kappa x)\right) \cos \left(\omega_{m} t\right),\right. \\
& \begin{array}{l}
\widetilde{u}_{2}^{m}(x, t)=-\frac{h_{1}^{0}}{h_{2}^{0}} \widetilde{u}_{1}^{m} \\
\widetilde{h}_{2}^{m}(x, t)=-\widetilde{h}_{1}^{m}(x, t)
\end{array}
\end{aligned}
$$

where $\widehat{q}_{m}$ and $G_{m}$ are arbitrary real numbers, $\kappa=2 \pi / L$, and

$$
\omega_{m}=m \kappa \sqrt{\frac{\left(\rho_{2}-\rho_{1}\right) g h_{1}^{0} h_{2}^{0}}{\rho_{1} h_{2}^{0}+\rho_{2} h_{1}^{0}}} .
$$

The resonance frequencies (2.27) can be expressed in terms of the two-fluid natural frequencies

$$
\omega_{j}^{\text {resonance }}=\frac{2}{L} \sqrt{\frac{\left(\rho_{2}-\rho_{1}\right) g h_{1}^{0} h_{2}^{0}}{\rho_{2} h_{1}^{0}+\rho_{1} h_{2}^{0}}} j \pi=2 \omega_{j}^{f}, \quad \text { for } \quad j \in \mathbb{N} .
$$

\section{Setting up the governing equations for numerics}

In solving the two-layer shallow water system numerically several difficulties arise. First, the system is conditionally hyperbolic. Secondly, an explicit expression cannot be found for the eigenvalues of the two layer system. Thirdly, a numerical scheme is required to be wellbalanced with source terms since the quasi-linear two layer shallow water system is nonconservative [28]. The eigenstructure of the hyperbolic PDEs (1.1) with its fourth equation replaced by (1.3) would be identical to two uncoupled shallow water equation systems [35] and it is shown in [15] that the approach of splitting of the layers would be unstable and suitable entropy fix is required [11]. The eigenvalues of the system (1.1) with the lower layer momentum equation replaced by (1.3) do not take into account the coupling between the layers and the eigenstructure of this system is not desirable for methods that need this information to construct a Riemann solution [35]. So the strategy is to use the two-layer shallow water system in (1.1) to determine the required eigenstructure for the f-wave finite volume analysis, and use the system (1.1) with the lower layer momentum equation replaced by (1.3) to calculate the jump in the fluxes.

The two-layer shallow water equations in the coupled system (1.1) can be written in the following balance law form

$$
\mathbf{q}(x, t)_{t}+f(\mathbf{q})_{x}=\Psi(\mathbf{q}, t)
$$


where

$$
\mathbf{q}(x, t)=\left[\begin{array}{c}
\rho_{1} h_{1} \\
\rho_{1} h_{1} u_{1} \\
\rho_{2} h_{2} \\
\rho_{2} h_{2} u_{2}
\end{array}\right], f(\mathbf{q})=\left[\begin{array}{c}
\rho_{1} h_{1} u_{1} \\
\rho_{1} h_{1} u_{1}^{2}+\frac{1}{2} \rho_{1} g h_{1}^{2} \\
\rho_{2} h_{2} u_{2} \\
\rho_{2} h_{2} u_{2}^{2}+\frac{1}{2} \rho_{2} g h_{2}^{2}
\end{array}\right], \Psi(\mathbf{q}, t)=\left[\begin{array}{c}
0 \\
-\rho_{1} g h_{1} h_{2 x}-h_{1} p_{x}-\rho_{1} h_{1} \ddot{q} \\
0 \\
-\rho_{1} g h_{2} h_{1 x}-h_{2} p_{x}-\rho_{2} h_{2} \ddot{q}
\end{array}\right] .
$$

This system can be written in quasi-linear form as

$$
\mathrm{q}_{t}+A(\mathrm{q}) \mathrm{q}_{x}=\widehat{\Psi}(\mathrm{q}, t)
$$

where

$$
A(\mathbf{q})=\left[\begin{array}{cccc}
0 & 1 & 0 & 0 \\
-u_{1}^{2}+g h_{1} & 2 u_{1} & r g h_{1} & 0 \\
0 & 0 & 0 & 1 \\
g h_{2} & 0 & -u_{2}^{2}+g h_{2} & 2 u_{2}
\end{array}\right], \quad \widehat{\Psi}(\mathbf{q}, t)=\left[\begin{array}{c}
0 \\
-h_{1} p_{x}-\rho_{1} h_{1} \ddot{q} \\
0 \\
-h_{2} p_{x}-\rho_{2} h_{2} \ddot{q}
\end{array}\right] .
$$

The characteristic polynomial of $A(\mathrm{q})$ reads

$$
\left(\left(\lambda-u_{2}\right)^{2}-g h_{2}\right)\left(\left(\lambda-u_{1}\right)^{2}-g h_{1}\right)-r g^{2} h_{1} h_{2}=0,
$$

where $r=\frac{\rho_{1}}{\rho_{2}}$. An interesting geometrical interpretation of $(3.3)$ is discussed in $[37,28,27]$.

\subsection{Computing the eigenvalues of the Jacobian}

Several approaches have been used in the literature to find the eigenvalues of the characteristic equation (3.3). In [29] an explicit expression for the roots of a fourth degree polynomial is used to evaluate the eigenvalues directly. This approach is appealing and it is being tested on the equations for the two-fluid system. However, numerically this direct approach is expensive and difficult to do with precision [35]. Therefore, in this paper, approximate methods are used to approximate the eigenvectors and eigenvalues.

In [41] a velocity difference expansion method is used to evaluate the eigenvalues. Approximations are based on an expansion about the differences in layer speeds $u_{1}-u_{2}$. Under the assumptions that $\left|u_{1}-u_{2}\right|$ and $(1-r)$ are very small, first order approximations for the eigenspeeds are

$$
\lambda_{\mathrm{ext}}^{ \pm} \approx \frac{h_{1} u_{1}+h_{2} u_{2}}{h_{1}+h_{2}} \pm \sqrt{g\left(h_{1}+h_{2}\right)}
$$

corresponding to wave speeds of surface waves and

$$
\lambda_{\mathrm{int}}^{ \pm} \approx \frac{h_{1} u_{2}+h_{2} u_{1}}{h_{1}+h_{2}} \pm \sqrt{g^{\prime} \frac{h_{1} h_{2}}{h_{1}+h_{2}}\left(1-\frac{\left(u_{1}-u_{2}\right)^{2}}{g^{\prime}\left(h_{1}+h_{2}\right)}\right)}
$$

where $g^{\prime}=g(1-r)$ is the reduced gravity, corresponding to wave speeds at the internal surface. So it can be concluded that the internal eigenvalues $\lambda_{\text {int }}^{ \pm}$are conditionally real if

$$
\frac{\left(u_{1}-u_{2}\right)^{2}}{g^{\prime}\left(h_{1}+h_{2}\right)}<1
$$


and it is expected when $\left|u_{1}-u_{2}\right|$ becomes large enough the shear stress grows and leads to the Kelvin-Helmholtz (KH) instability. So this condition is linked to the KH instability of the stratified flows [28, 27]. See [14] for the numerical treatment of the loss of hyperbolicity of the two layer shallow water system, and see [10] for the nonlinear stability analysis of twolayer shallow water equations with a rigid-lid. This approach for the approximation of the eigenvalues is useful only for two-layer shallow fluid flows with densities very close to each other (Boussinesq approximation). In this paper we are interested in two-layer shallow fluid flows with $r$ small (non-Boussinesq limit). Hence, we adapt the following approach for the approximation of the eigenvalues.

Another approach to approximating the eigenstructure of (1.1) is to use the eigenvalues of the linearized system with the characteristic polynomial

$$
\left(\lambda^{2}-g h_{2}^{0}\right)\left(\lambda^{2}-g h_{1}^{0}\right)-r g^{2} h_{1}^{0} h_{2}^{0}=0,
$$

where $h_{1}^{0}$ and $h_{2}^{0}$ are the upper layer and lower layer fluid depth at rest, respectively. Assuming that the eigenvectors have the form $\widetilde{\mathrm{x}}=[1, \lambda, \chi, \lambda \chi]^{T}$, the unknowns $\lambda$ and $\chi$ can be found from $A \widetilde{\mathrm{x}}=\lambda \widetilde{\mathrm{x}}$, where $A$ is defined in (2.2),

$$
g h_{1}^{0}(1+\chi r)=\lambda^{2}, \quad g h_{2}^{0}(1+\chi)=\lambda^{2} \chi .
$$

Now eliminating $\lambda$ from both equations, a quadratic equation for $\chi$ can be found

$$
\chi^{2}+\frac{1}{r}(1-\beta) \chi-\frac{\beta}{r}=0
$$

giving

$$
2 \chi^{ \pm}=-\frac{1}{r}(1-\beta) \pm \frac{1}{r} \sqrt{(1-\beta)^{2}+4 \beta r},
$$

where $\beta=\frac{h_{2}^{0}}{h_{1}^{0}}$. Now $\lambda$ can be found in terms of either the top or bottom layers,

$$
\lambda= \pm \sqrt{g h_{1}^{0}} \sqrt{1+\chi r}, \quad \lambda= \pm \sqrt{g h_{2}^{0}} \sqrt{\frac{1+\chi}{\chi}},
$$

and it is discussed in $[35,28,27]$ that the external and internal eigenvalues are

$$
\begin{aligned}
& \lambda_{1}^{\text {ext }}=-\sqrt{g h_{1}^{0}\left(1+\chi^{+} r\right)}, \quad \lambda_{1}^{\text {int }}=-\sqrt{g h_{1}^{0}\left(1+\chi^{-} r\right)}, \\
& \lambda_{2}^{\text {int }}=\sqrt{g h_{1}^{0}\left(1+\chi^{-} r\right)}, \quad \lambda_{2}^{\text {ext }}=\sqrt{g h_{1}^{0}\left(1+\chi^{+} r\right)},
\end{aligned}
$$

with eigenvectors

$$
\Lambda=\left[\begin{array}{cccc}
1 & 1 & 1 & 1 \\
-\sqrt{g h_{1}^{0}\left(1+\chi^{+} r\right)} & -\sqrt{g h_{1}^{0}\left(1+\chi^{-} r\right)} & \sqrt{g h_{1}^{0}\left(1+\chi^{-} r\right)} & \sqrt{g h_{1}^{0}\left(1+\chi^{+} r\right)} \\
\chi^{+} & \chi^{-} & \chi^{-} & \chi^{+} \\
-\chi^{+} \sqrt{g h_{1}^{0}\left(1+\chi^{+} r\right)} & -\chi^{-} \sqrt{g h_{1}^{0}\left(1+\chi^{-} r\right)} & \chi^{-} \sqrt{g h_{1}^{0}\left(1+\chi^{-} r\right)} & \chi^{+} \sqrt{g h_{1}^{0}\left(1+\chi^{+} r\right)}
\end{array}\right] .
$$

Since the eigenspace is completely determined by the initial conditions in the linear limit and does not change in time, an alternative to this is to use the full values of $\beta, h_{1}$ and $h_{2}$ for the nonlinear equations [34]. This latter approach is the one we use in this paper to approximate the eigenstructure of the two-layer shallow water system. For the nonlinear problem, $h_{1}^{0}$ and $h_{2}^{0}$ are replaced by the computed values of $h_{1}$ and $h_{2}$. 


\section{F-wave numerical approach for the fluid motion}

The numerical method used in this paper to implement the linear and nonlinear two layer shallow water equations with a rigid-lid is the high resolution wave propagation finite volume algorithm developed by BALE ET AL. [9, 31] and used by [22, 35, 27]. This method is briefly discussed here. Interested readers are referred to [9, 31, 21, 32, 22, 34, 35, 28, 27]. The wave propagation algorithm is Godunov type finite volume method often referred as REA algorithm, standing for reconstruction-evolve-average, making use of Riemann problems to determine the numerical update at each time step. Godunov's method uses the Riemann solutions to evaluate cell interface fluxes at each time step. In LeVeque's wave propagation algorithm the waves arising in Riemann solutions are re-averaged onto adjacent grid cells in order to update the numerical solution [22]. LeVeque's method is applicable to hyperbolic systems of the form (3.2). The solution to the Riemann problem consists of $m$ waves denoted by $\mathcal{W}^{p} \in \mathbb{R}^{\mathrm{m}}$ propagating out from each grid cell interface at speeds $s^{p}$. These waves are related to the jump discontinuity at each grid cell interface via

$$
\mathrm{Q}_{i}-\mathrm{Q}_{i-1}=\sum_{p=1}^{\mathrm{m}} \mathcal{W}_{i-1 / 2}^{p}=\sum_{p=1}^{\mathrm{m}} \alpha_{i-1 / 2}^{p} r_{i-1 / 2}^{p},
$$

where $r_{i-1 / 2}^{p}$ are eigenvectors of the approximate flux Jacobian $\widehat{A}_{i-1 / 2}$ and $\mathrm{Q}_{i}^{n}=\frac{1}{\Delta x} \int_{\mathcal{C}_{i}} \mathrm{q}\left(x, t^{n}\right) d x$ with $\mathcal{C}_{i}=\left[x_{i-1 / 2}, x_{i+1 / 2}\right], \Delta x=\left(x_{i+1 / 2}-x_{i-1 / 2}\right)$ and $\Delta t=\left(t^{n+1}-t^{n}\right)$ and the domain is partitioned into grid cells $\mathcal{C}$. This amounts to a projection of the jump in $\mathrm{Q}$ onto the eigenspace of $\widehat{A}_{i-1 / 2}$. The first order upwind method then reads

$$
\mathrm{Q}_{i}^{n+1}=\mathrm{Q}_{i}^{n}-\frac{\Delta t}{\Delta x}\left(\mathcal{A}^{+} \Delta \mathrm{Q}_{i-1 / 2}+\mathcal{A}^{-} \Delta \mathrm{Q}_{i+1 / 2}\right)
$$

The fluctuations $\mathcal{A}^{ \pm} \Delta \mathrm{Q}_{i \mp 1 / 2}^{n}$ are determined by solutions to Riemann problems at the cell interfaces at $x_{i \pm 1 / 2}$. The term $\mathcal{A}^{+} \Delta \mathrm{Q}_{i-1 / 2}$ represents the net updating contribution from the rightward moving waves into grid cell $\mathcal{C}_{i}$ from the left interface, and $\mathcal{A}^{-} \Delta \mathrm{Q}_{i+1 / 2}$ represents the net updating contribution from the leftward moving waves into cell $\mathcal{C}_{i}$ from the right interface. These fluctuations can be defined in terms of waves as

$$
\mathcal{A}^{ \pm} \Delta \mathrm{Q}_{i-1 / 2}=\sum_{p=1}^{\mathrm{m}}\left(s_{i-1 / 2}^{p}\right)^{ \pm} \mathcal{W}_{i-1 / 2}^{p},
$$

where $s_{i-1 / 2}^{+}=\max \left(s_{i-1 / 2}^{p}, 0\right)$ and $s_{i-1 / 2}^{-}=\min \left(s_{i-1 / 2}^{p}, 0\right)$. The wave propagation method (4.2) can be extended to second order accuracy using limiters applied to each wave such that

$$
\mathrm{Q}_{i}^{n+1}=\mathrm{Q}_{i}^{n}-\frac{\Delta t}{\Delta x}\left(\mathcal{A}^{+} \Delta \mathrm{Q}_{i-1 / 2}+\mathcal{A}^{-} \Delta \mathrm{Q}_{i+1 / 2}\right)-\frac{\Delta t}{\Delta x}\left(\widetilde{F}_{i+1 / 2}-\widetilde{F}_{i-1 / 2}\right),
$$

where

$$
\widetilde{F}_{i-1 / 2}=\frac{1}{2} \sum_{p=1}^{m}\left|s_{i-1 / 2}^{p}\right|\left(1-\frac{\Delta t}{\Delta x}\left|s_{i-1 / 2}^{p}\right|\right) \widetilde{\mathcal{W}}_{i-1 / 2}^{p},
$$

where $\widetilde{\mathcal{W}}_{i-1 / 2}^{p}$ are limited versions of $\mathcal{W}_{i-1 / 2}^{p}$. There are different standard limiter functions that ensure TVD stability of the solution (see [31,22]). A common choice for the pair $\left\{r_{i-1 / 2}^{p}, s_{i-1 / 2}^{p}\right\}$ 
is the $p$ th eigenpair of a local linear approximation to the flux Jacobian matrix $\widehat{A}(\mathrm{q})$ at $x_{i-1 / 2}$ such as Roe averaging.

A consistent alternative approach to the wave propagation method (4.1) for a conservation law is to decompose the jump in fluxes into waves instead of the states q such that

$$
f\left(\mathrm{Q}_{i}\right)-f\left(\mathrm{Q}_{i-1}\right)=\sum_{p=1}^{\mathrm{m}} \mathcal{Z}_{i-1 / 2}^{p}=\sum_{p=1}^{\mathrm{m}} \beta_{i-1 / 2}^{p} r_{i-1 / 2}^{p},
$$

where the waves $\mathcal{Z}_{i-1 / 2}^{p}=\beta_{i-1 / 2}^{p} r_{i-1 / 2}^{p}$ are called $f$-waves and represent propagating jumps in the flux and similar to the $\mathcal{W}$ s we have $\mathcal{Z}^{p} \in \mathbb{R}^{\mathrm{m}}$. Fluctuations are therefore defined by

$$
\mathcal{A}^{ \pm} \Delta \mathrm{Q}_{i-1 / 2}=\sum_{p=1}^{\mathrm{m}} \operatorname{sgn}\left(s_{i-1 / 2}^{p}\right) \mathcal{Z}_{i-1 / 2}^{p} .
$$

The higher order correction terms are given by

$$
\widetilde{F}_{i-1 / 2}=\frac{1}{2} \sum_{p=1}^{m} \operatorname{sgn}\left(s_{i-1 / 2}^{p}\right)\left(1-\frac{\Delta t}{\Delta x}\left|s_{i-1 / 2}^{p}\right|\right) \widetilde{\mathcal{Z}}_{i-1 / 2}^{p},
$$

where $\widetilde{\mathcal{Z}}_{i-1 / 2}^{p}$ is a limited version of the f-wave $\mathcal{Z}_{i-1 / 2}^{p}$ using a TVD limiter [31].

The advantage of using the f-wave method over the wave-propagation method is that it is conservative regardless of the linearization used for the flux Jacobian to calculate the eigenspace. It also extends to spatially varying flux terms $\Psi(\mathrm{q}, x)$. The advantage that we will make the most use of in solving the two-layer shallow water equations with pressure gradient at the rigid-lid is the ability to use source terms to modify the flux difference before doing wave decomposition such that

$$
\delta=f\left(\mathrm{Q}_{i}\right)-f\left(\mathrm{Q}_{i-1}\right)-\Delta x \Psi_{i-1 / 2}=\sum_{p=1}^{\mathrm{m}} \mathcal{Z}_{i-1 / 2}^{p},
$$

for some representation of the source term $\Psi$ at $x_{i-1 / 2}$ such that

$$
\Delta x \Psi_{i-1 / 2} \approx \int \Psi(\mathrm{q}, x) d x
$$

The basic steps in implementing the f-wave-propagation method are the evaluation of the relevant states to the Riemann problem using the vectors $Q_{i}$ and $Q_{i-1}$, computation of the eigenvalues and eigenvectors $\left(s^{p}, r^{p}\right)$, computation of the jump in the fluxes and source terms $\delta$, projection of $\delta$ onto the eigenspace to determine the f-waves $\mathcal{Z}^{p}$, and finally calculation of the fluctuations $\mathcal{A}^{ \pm} \Delta \mathrm{Q}$ [35]. For most of the simulations in this paper the eigenstructure of the linearized equations is used as a basis for the approximation of the eigenvalues and eigenvectors of the nonlinear system as is briefly discussed in $§ 3$. See $[34,35]$ for more details.

Pressure gradient appears as a time-dependent source term in the finite volume formulation of the two-fluid equations. See $\widehat{\Psi}(\mathrm{q}, t)$ in (3.2), and $\widehat{\Psi}(\widetilde{\mathrm{q}}, t)$ in (2.2) for the nonlinear and linear two-layer shallow-water equations, respectively. For the numerical simulations in this paper, the exact expression for the pressure gradient at the rigid-lid is included into the numerical calculations. For the linear equations, write (2.5) in the following form

$$
\frac{\partial \widetilde{p}}{\partial x}=\frac{\rho_{1} \rho_{2}}{\rho_{2} h_{1}^{0}+\rho_{1} h_{2}^{0}}\left(-g h_{2}^{0} \frac{\partial \widetilde{h}_{2}}{\partial x}-r g h_{2}^{0} \frac{\partial \widetilde{h}_{1}}{\partial x}\right)-\frac{\rho_{1} \rho_{2}}{\rho_{2} h_{1}^{0}+\rho_{1} h_{2}^{0}} d \ddot{\widetilde{q}} .
$$


The first part would be considered in the jump in the flux vectors $\delta$ in (4.5) before decomposing the differences into waves propagating out from each grid cell interface. The resulting differences are

$$
\delta=\left[\begin{array}{c}
{\left[\rho_{1} h_{1}^{0} \widetilde{u}_{1}\right]} \\
{\left[\rho_{1} g h_{1}^{0} \widetilde{h}_{1}\right]+\left[\rho_{1} g h_{1}^{0} \widetilde{h}_{2}\right]+h_{1}^{0} \mathrm{~A}} \\
{\left[\rho_{2} h_{2}^{0} \widetilde{u}_{2}\right]} \\
{\left[\rho_{2} g h_{2}^{0} \widetilde{h}_{2}+\rho_{1} g h_{1}^{0} \widetilde{h}_{2}+\rho_{1} g h_{2}^{0} \widetilde{h}_{1}\right]-\left[\rho_{1} g h_{1}^{0} \widetilde{h}_{2}\right]+h_{2}^{0} \mathrm{~A}}
\end{array}\right]
$$

where [.] represents the state differences across the cell interface and

$$
\mathrm{A}=\frac{\rho_{1} \rho_{2}}{\rho_{2} h_{1}^{0}+\rho_{1} h_{2}^{0}}\left(-g h_{2}^{0}\left[\widetilde{h}_{2}+r \widetilde{h}_{1}\right]\right) .
$$

The time-dependent source terms due to the acceleration of the vessel motion are handled via a fractional step approach such that after each time step of the hyperbolic problem a time step is taken in which the momentum equations are adjusted due to the forcing terms. Given $\widetilde{h}_{1}, \widetilde{h}_{2}, \widetilde{u}_{1}$ and $\widetilde{u}_{2}$ at time level $n$, apply the f-wave-propagation finite volume method to update these over time $\Delta t$ by solving the inhomogeneous linear hyperbolic system (2.2) and call the new values $\widetilde{h}_{1}^{\star}, \widetilde{h}_{2}^{\star}, \widetilde{u}_{1}^{\star}$ and $\widetilde{u}_{2}^{\star}$. Then update the star values to $\widetilde{h}_{1}^{n+1}, \widetilde{h}_{2}^{n+1}, \widetilde{u}_{1}^{n+1}$ and $\widetilde{u}_{2}^{n+1}$ by solving the equations

$$
\left.\begin{array}{rl}
\frac{\partial}{\partial t}\left(\rho_{1} \widetilde{h}_{1}\right) & =0, \\
\frac{\partial}{\partial t}\left(\rho_{1} h_{1}^{0} \widetilde{u}_{1}\right) & =\frac{h_{1}^{0} \rho_{1} \rho_{2}}{\rho_{2} h_{1}^{0}+\rho_{1} h_{2}^{0}} d \ddot{\widetilde{q}}-\rho_{1} h_{1}^{0} \ddot{\widetilde{q}}, \\
\frac{\partial}{\partial t}\left(\rho_{2} \widetilde{h}_{2}\right) & =0, \\
\frac{\partial}{\partial t}\left(\rho_{2} h_{2}^{0} \widetilde{u}_{2}\right) & =\frac{h_{2}^{0} \rho_{1} \rho_{2}}{\rho_{2} h_{1}^{0}+\rho_{1} h_{2}^{0}} d \ddot{\widetilde{q}}-\rho_{2} h_{2}^{0} \ddot{\widetilde{q}} .
\end{array}\right\}
$$

For the nonlinear hyperbolic system (3.2), differentiate the constraint equation $h_{1}(x, t)+h_{2}(x, t)=$ $d$ two times with respect to time and use the mass and momentum equations to obtain the exact expression for the pressure gradient at the rigid-lid,

$$
p_{x}=\frac{\rho_{1} \rho_{2}}{\rho_{2} h_{1}+\rho_{1} h_{2}}\left(-\left(h_{1} u_{1}^{2}+\frac{1}{2} g h_{1}^{2}+h_{2} u_{2}^{2}+\frac{1}{2} g h_{2}^{2}+r g h_{1} h_{2}\right)_{x}-g^{\prime} h_{1} h_{2 x}-d \ddot{q}\right) .
$$

Similar to the linear problem, the jump in the flux vectors $\delta$ can be computed as

$$
\delta=\left[\begin{array}{c}
{\left[\rho_{1} h_{1} u_{1}\right]} \\
{\left[\rho_{1} h_{1} u_{1}^{2}+\frac{1}{2} \rho_{1} g h_{1}^{2}\right]+\rho_{1} g \bar{h}_{1}\left[h_{2}\right]+\bar{h}_{1} \mathrm{~B}} \\
{\left[\rho_{2} h_{2} u_{2}\right]} \\
{\left[\rho_{2} h_{2} u_{2}^{2}+\frac{1}{2} \rho_{2} g h_{2}^{2}+\rho_{1} g h_{1} h_{2}\right]-\rho_{1} g \bar{h}_{1}\left[h_{2}\right]+\bar{h}_{2} \mathrm{~B}}
\end{array}\right],
$$

with

$$
\mathrm{B}=\frac{\rho_{1} \rho_{2}}{\rho_{2} \bar{h}_{1}+\rho_{1} \bar{h}_{2}}\left(-\left[h_{1} u_{1}^{2}+\frac{1}{2} g h_{1}^{2}+h_{2} u_{2}^{2}+\frac{1}{2} g h_{2}^{2}+r g h_{1} h_{2}\right]-g^{\prime} \bar{h}_{1}\left[h_{2}\right]\right),
$$

where [.] and - represents the difference and average across the cell interface, respectively. Averages are used for layer depths as motivated by the path-conservative jump conditions assuming 
a linear path through state space (see [34, 35]). For the time-dependent source terms use the fractional step approach and update the intermediate state values by solving the equations

$$
\left.\begin{array}{rl}
\frac{\partial}{\partial t}\left(\rho_{1} h_{1}\right) & =0, \\
\frac{\partial}{\partial t}\left(\rho_{1} h_{1} u_{1}\right) & =\frac{\rho_{1} \rho_{2} h_{1}}{\rho_{2} h_{1}+\rho_{1} h_{2}} d \ddot{q}-\rho_{1} h_{1} \ddot{q}, \\
\frac{\partial}{\partial t}\left(\rho_{2} h_{2}\right) & =0, \\
\frac{\partial}{\partial t}\left(\rho_{2} h_{2} u_{2}\right) & =\frac{\rho_{1} \rho_{2} h_{2}}{\rho_{2} h_{1}+\rho_{1} h_{2}} d \ddot{q}-\rho_{2} h_{2} \ddot{q} .
\end{array}\right\}
$$

\section{Numerical approach for the vessel motion}

The governing equation for the motion of the vessel with interior two-fluid sloshing is a secondorder ODE, forced by the infinite-dimensional fluid motion. For the linear coupled system the vessel equation is

$$
M \frac{d^{2} \widetilde{q}}{d t^{2}}+\frac{d}{d t} \int_{0}^{L}\left(\rho_{1} h_{1}^{0} \widetilde{u}_{1}+\rho_{2} h_{2}^{0} \widetilde{u}_{2}\right) d x+\nu \widetilde{q}=0
$$

where $M=m_{f}^{1}+m_{f}^{2}+m_{v}$. This equation can be written as a system of first-order ODEs. Setting

$$
\gamma_{1}=\widetilde{q}, \quad \gamma_{2}=\dot{\widetilde{q}}
$$

then

$$
\left.\begin{array}{l}
\dot{\gamma}_{1}=\gamma_{2}, \\
\dot{\gamma}_{2}=-\frac{\nu}{M} \gamma_{1}-\frac{\rho_{1} h_{1}^{0}}{M} \int_{0}^{L} \frac{\partial \widetilde{u}_{1}}{\partial t} d x-\frac{\rho_{2} h_{2}^{0}}{M} \int_{0}^{L} \frac{\partial \widetilde{u}_{2}}{\partial t} d x .
\end{array}\right\}
$$

The strategy is to solve the linear two-layer shallow-water equations (2.2) with the f-wavepropagation finite volume method explicitly, and then evaluate the right-hand side integrals in (5.3) using a quadrature formula. Then the system of first-order ODEs (5.3) can be solved with different time integration methods. In this paper we use the MATLAB ODE45 built in explicit Runge-Kutta solver for the time integration.

For the nonlinear coupled system, the vessel equation is

$$
M \frac{d^{2} q}{d t^{2}}+\frac{d}{d t} \int_{0}^{L}\left(\rho_{1} h_{1} u_{1}+\rho_{2} h_{2} u_{2}\right) d x+\nu_{1} q-\nu_{2} q^{3}=0 .
$$

This equation can be written as a system of first-order ODEs. Setting

$$
\Gamma_{1}=q, \quad \Gamma_{2}=\dot{q},
$$

then

$$
\left.\begin{array}{l}
\dot{\Gamma}_{1}=\Gamma_{2}, \\
\dot{\Gamma}_{2}=-\frac{\nu_{1}}{M} \Gamma_{1}+\frac{\nu_{2}}{M} \Gamma_{1}^{3}-\frac{1}{M} \int_{0}^{L} \frac{\partial}{\partial t}\left(\rho_{1} h_{1} u_{1}\right) d x-\frac{1}{M} \int_{0}^{L} \frac{\partial}{\partial t}\left(\rho_{2} h_{2} u_{2}\right) d x .
\end{array}\right\}
$$


Similarly for the nonlinear coupled problem, the two-layer shallow-water equations (3.2) are solved explicitly with the f-wave-propagation finite volume method and then the right-hand side integrals in (5.6) are evaluated with a quadrature formula, and the system of first-order ODEs (5.6) are solved using the MATLAB ODE45 routine.

\section{Results}

Numerical results are divided into two parts. In $\S 6.1$ the results of linear numerical simulations against the exact linear solutions are reported in order to validate the coupled dynamic coupling solver. The linear simulations are presented for the case of internal $1: 1$ resonance in the Boussinesq and non-Boussinesq limits where the interface profile resembles a standing wave with three nodes, and for the root $s_{2}$ of the characteristic equation with $\nu<\nu_{1: 1}$ in the Boussinesq limit where the interface profile resembles a standing wave with one node, and also for the root $s_{1}$ of the characteristic equation in the non-Boussinesq limit where the interface profile resembles a standing wave with one node which oscillates in phase with the vessel motion. A comparison of the natural frequencies of the coupled and uncoupled systems is also given in this section.

In $\S 6.2$ the results of nonlinear numerical simulations are presented for different cases where the nonlinear two-fluid equations are coupled to the linear and nonlinear vessel equation with various spring constants including the case of internal $1: 1$ resonances.

\subsection{Linear simulations}

The first simulations are based on the exact oscillatory solutions derived in $\$ 2$ with rigid wall boundary conditions.

The first simulation is for the case of internal $1: 1$ resonance, when $G=m^{2} \pi^{2} R$. The most interesting case is for $m=1$, so we set the spring constant to $\nu=\nu_{1: 1}$ as derived in (2.25) with $m=1$. Take the input parameters as

$$
\begin{aligned}
\rho_{1} & =900 \mathrm{~kg} / \mathrm{m}^{3}, \quad \rho_{2}=1000 \mathrm{~kg} / \mathrm{m}^{3}, \quad L=1 \mathrm{~m}, \quad \nu=\nu_{1: 1}=67.9902 \mathrm{~kg} / \mathrm{sec}^{2}, \\
h_{1}^{0} & =.04 \mathrm{~m}, \quad h_{2}^{0}=.04 \mathrm{~m}, \quad \widehat{q}=.22 \mathrm{~m}, \quad \Delta x=.01 \mathrm{~m}, \quad \Delta t=.001 \mathrm{sec}, \\
m_{f}^{1} & =36 \mathrm{~kg}, \quad m_{f}^{2}=40 \mathrm{~kg}, \quad m_{v}=.1\left(m_{f}^{1}+m_{f}^{2}\right)=7.6 \mathrm{~kg}, \quad g=9.81 \mathrm{~m} / \mathrm{sec}^{2}, \\
\mathrm{R} & =396.10, \quad \mathrm{G}=3909.35, \quad \omega^{\text {coupled }}=\omega_{1}^{\text {resonance }}=.9029 \mathrm{rad} / \mathrm{sec}, \quad T^{\text {coupled }}=6.958 \mathrm{sec} .
\end{aligned}
$$

Figure 2 shows the snapshots of the computed (solid lines) and exact wave profiles (dashed lines). The numerical solutions are in good agreement with the exact solutions.

For the dynamic coupling problem with one-layer, the root $s_{1}$ corresponds to the longest wavelength, for which the vessel and wave motions are in phase: as the vessel moves to the right, the interface at the right-hand end of the vessel rises until maximum run-up coincides with the time of extreme displacement of the vessel to the right, and the interface at the left-hand end rises during the leftward movement of the vessel [16]. Also for the one-layer dynamic coupling problem, the root $s_{2}$ corresponds to a range of surface shapes with between one and three nodes 

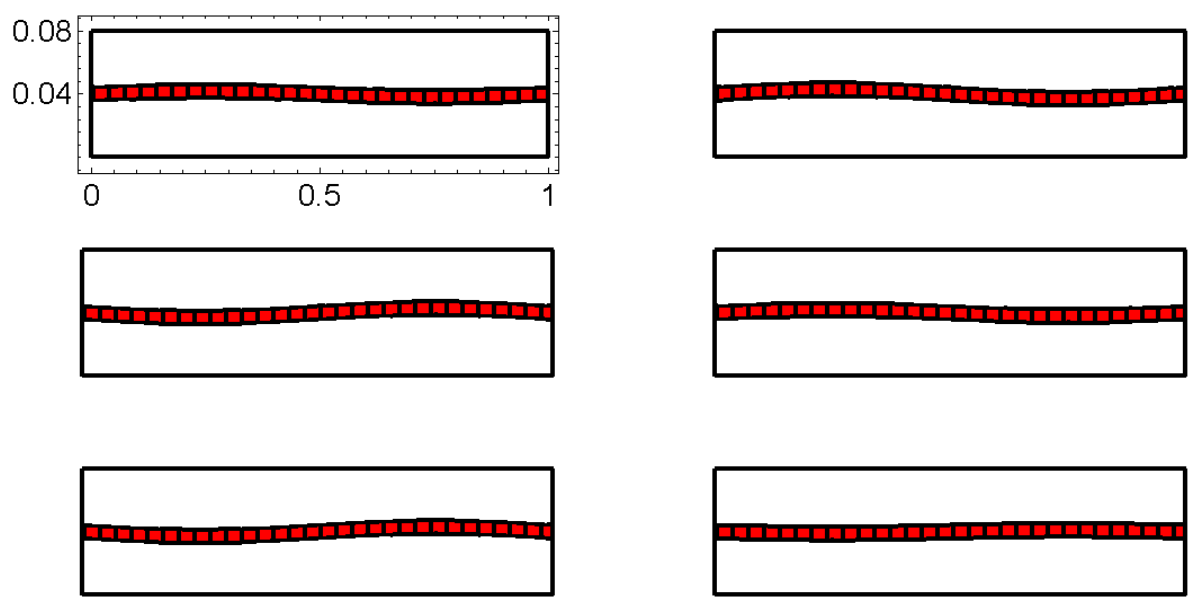

Figure 2: (colour online) Snapshots of the interface profile at $t=1 \mathrm{~s}, t=7 \mathrm{~s}$ for the first row from left to right, $t=10.4 \mathrm{~s}, t=20 \mathrm{~s}$ for the second row from left to right, and $t=24.5 \mathrm{~s}$, $t=30 \mathrm{~s}$ for the third row from left to right. The horizontal and vertical axes are, respectively, $x(m)$ and $h_{2,1}(x, t)$. The numerical solution is shown in solid line and the exact solution in dashed red line.

[16]. If there is one node then the wave oscillates out of phase (antiphase) with the vessel motion.

For the two-fluid configuration here the snapshots of the computed wave profile at integer and half integer multiples of the coupled natural frequency $T^{\text {coupled }}=6.958 \mathrm{sec}$, and also at $t=$ $1 \mathrm{sec}$, and $t=20 \mathrm{sec}$ are shown in Figure 3. The interface profile resembles a standing wave with three nodes.

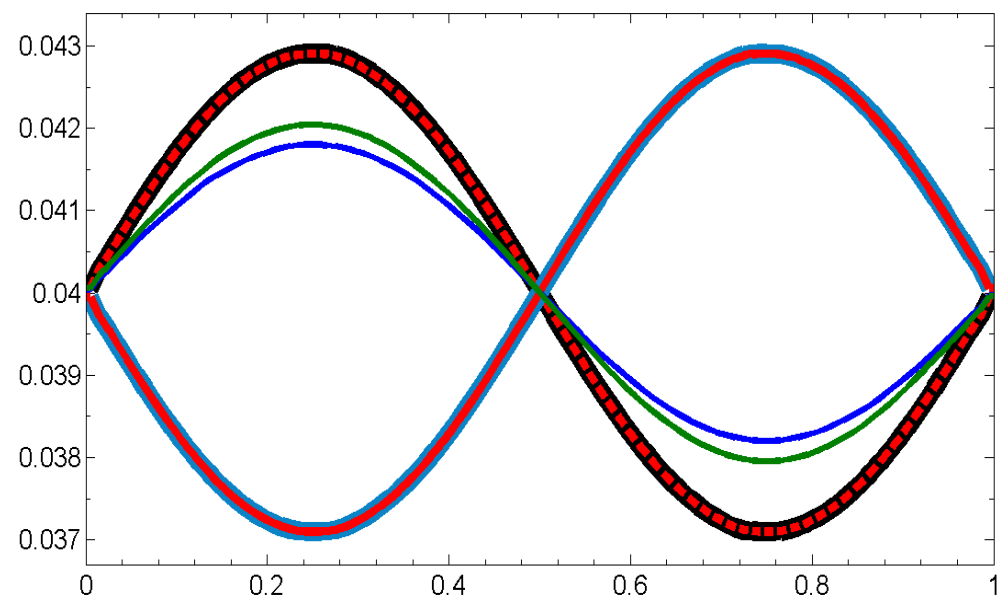

Figure 3: (colour online) Standing waves with three nodes at $1: 1$ resonance. Snapshots of the computed (numerical solutions) interface profile at $t=6.958 \mathrm{sec}$ in solid black line, at $t=4 \times 6.958 \mathrm{sec}$ in dashed red line, at $t=\frac{1}{2} \times 6.958 \mathrm{sec}$ in solid light blue line, at $t=$ $\frac{1}{2} \times 6.958+3 \times 6.958 \mathrm{sec}$ in solid red line, at $t=1 \mathrm{sec}$ in dark blue line, and at $t=20 \mathrm{sec}$ in green line. 
With no flow boundary conditions the constraint equation is

$$
h_{1}^{0} \widetilde{u}_{1}+h_{2}^{0} \widetilde{u}_{2}=0 .
$$

The first row in Figure 4 shows $h_{1}^{0} \widetilde{u}_{1}+h_{2}^{0} \widetilde{u}_{2}$ at $t=30 \mathrm{sec}$, the second row shows the conservation of the rigid-lid constraint $\widetilde{h}_{1}+\widetilde{h}_{2}=0$ at $x=.5 \mathrm{~m}$ versus time, and the third row shows $h_{1}^{0} \widetilde{u}_{1}+h_{2}^{0} \widetilde{u}_{2}=0$ at $x=.5 \mathrm{~m}$ versus time. The system constraints are preserved approximately with an error of order $\Delta t^{p}$ with $2<p<3$. Numerical errors are bounded over time. Figure 5
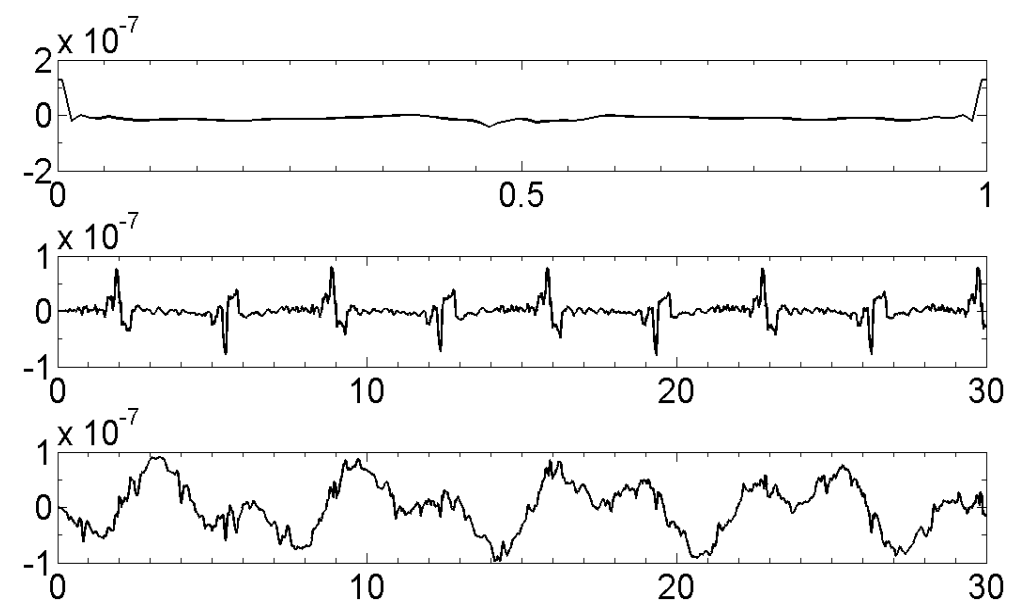

Figure 4: First row: $h_{1}^{0} \widetilde{u}_{1}+h_{2}^{0} \widetilde{u}_{2}$ at $t=30$ sec. Second row: $\widetilde{h}_{1}+\widetilde{h}_{2}$ at $x=.5 m$ versus time. Third row: $h_{1}^{0} \widetilde{u}_{1}+h_{2}^{0} \widetilde{u}_{2}$ at $x=.5 m$ versus time.

shows the numerical and exact solutions for the vessel displacement versus time which are in very good agreement.

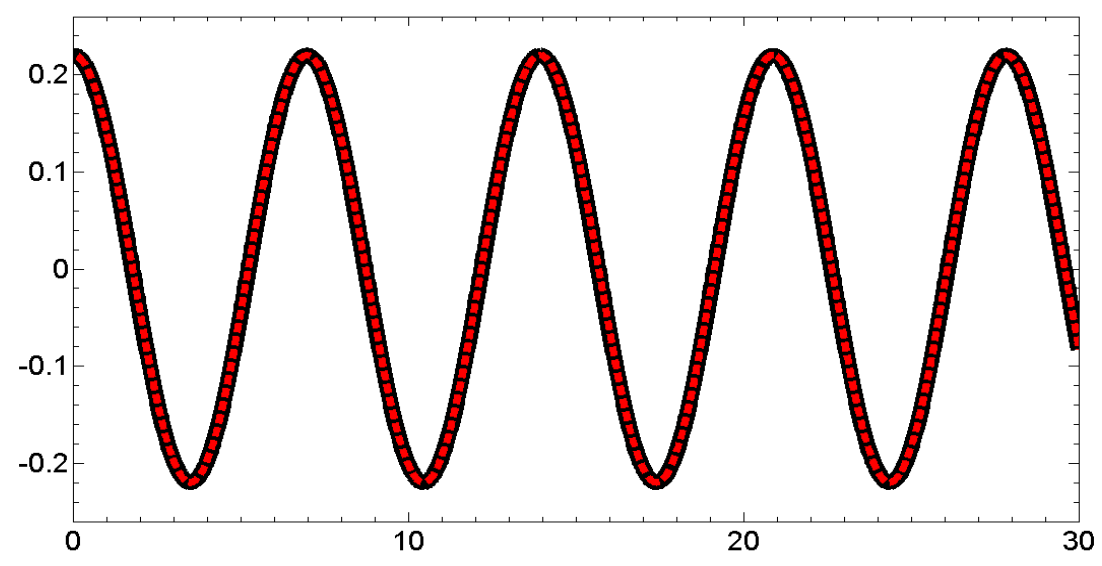

Figure 5: (colour online) Numerical and exact solutions for the vessel motion. The numerical solution is shown in solid line and the exact solution in dashed red line. The vertical axis is $q(m)$ and the horizontal axis is time.

For the coupled problem the vessel energy is defined by

$$
E_{v}=\frac{1}{2} m_{v} \dot{q}^{2}+\frac{1}{2} \nu q^{2},
$$


and the fluid energy is defined by

$$
E_{f}=\int_{0}^{L}\left(\frac{1}{2} \rho_{1} h_{1}\left(u_{1}+\dot{q}\right)^{2}+\frac{1}{2} \rho_{1} g h_{1}^{2}\right) d x+\int_{0}^{L}\left(\frac{1}{2} \rho_{2} h_{2}\left(u_{2}+\dot{q}\right)^{2}+\frac{1}{2} \rho_{2} g h_{2}^{2}\right) d x .
$$

The first row in Figure 6 shows the energy of the fluid versus time, and the second row shows the energy of the vessel versus time.
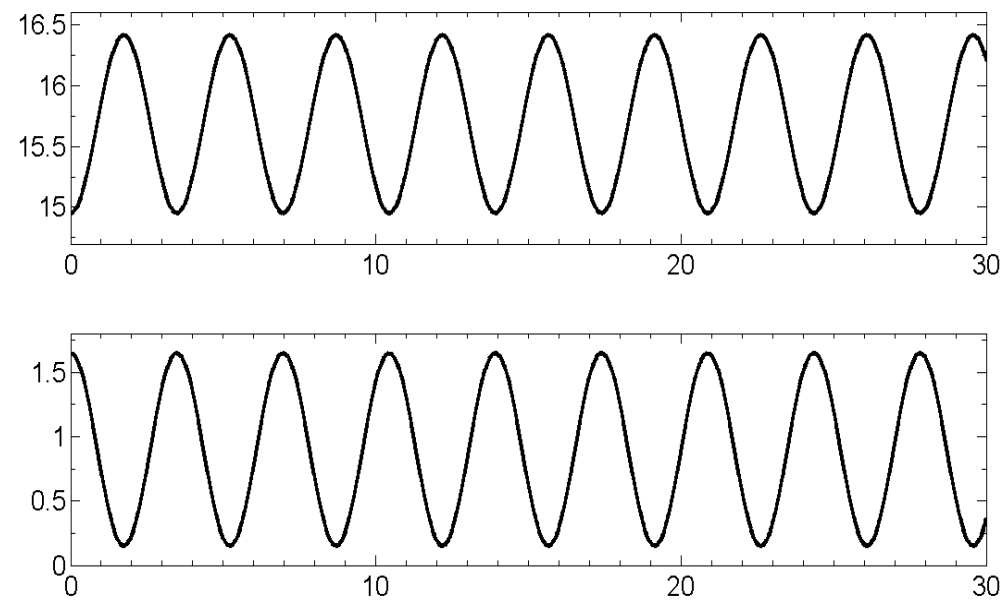

Figure 6: First row: fluid energy versus time. Second row: vessel energy versus time.

The second simulation is for the case of $\nu<\nu_{1: 1}$. Take the input parameters the same as the first simulation but with $\nu=.8 \nu_{1: 1}=54.3921 \mathrm{~kg} / \mathrm{sec}^{2}$. Numerical experiments show that the coupled natural frequency of a two-fluid dynamic coupling problem in the Boussinesq regime is greater than $\frac{1}{2} \pi$ and so the coupled system oscillates at higher modes. The natural frequencies and linear solution parameters for this example are

$$
\begin{aligned}
& \omega_{2}^{\text {coupled }}=.8077 \mathrm{rad} / \mathrm{sec}, \quad \omega_{1}^{f}=.4514 \mathrm{rad} / \mathrm{sec}, \quad \omega_{f v}=.8066 \mathrm{rad} / \mathrm{sec}, \\
& T^{\text {coupled }}=7.778 \mathrm{sec}, \quad s_{2}=2.81, \quad \mathrm{R}=396.10, \quad \mathrm{G}=3127.48 .
\end{aligned}
$$

Figure 7 shows the snapshots of the computed and exact wave profiles up to $32 \mathrm{sec}$. The numerical and exact solutions are in very good agreement. The first row in Figure 8 shows the numerical and exact solutions for the vessel displacement versus time up to $32 \mathrm{sec}$. The numerical and exact solutions are in very good agreement. The second row in Figure 8 shows the energy of the fluid (6.2) versus time, and the third row shows the energy of the vessel (6.1) versus time. The partition of energy between the fluid and vessel motion is maintained.

The computed numerical solutions for the interface wave profile for the root $s_{2}$ at integer and half integer multiples of the coupled natural frequency $T^{\text {coupled }}=7.778 \mathrm{sec}$ are depicted in Figure 9. The interface profile resembles a standing wave with one node for the root $s_{2}$ of the coupled oscillations with $\nu<\nu_{1: 1}$.

Numerical errors in preservation of the two constraints are shown in Figure 10. The first row in Figure 10 shows $h_{1}^{0} \widetilde{u}_{1}+h_{2}^{0} \widetilde{u}_{2}$ at $t=32 \mathrm{sec}$, the second row shows the conservation of the rigidlid constraint $\widetilde{h}_{1}+\widetilde{h}_{2}=0$ at $x=.5 m$ versus time, and the third row shows $h_{1}^{0} \widetilde{u}_{1}+h_{2}^{0} \widetilde{u}_{2}=0$ 

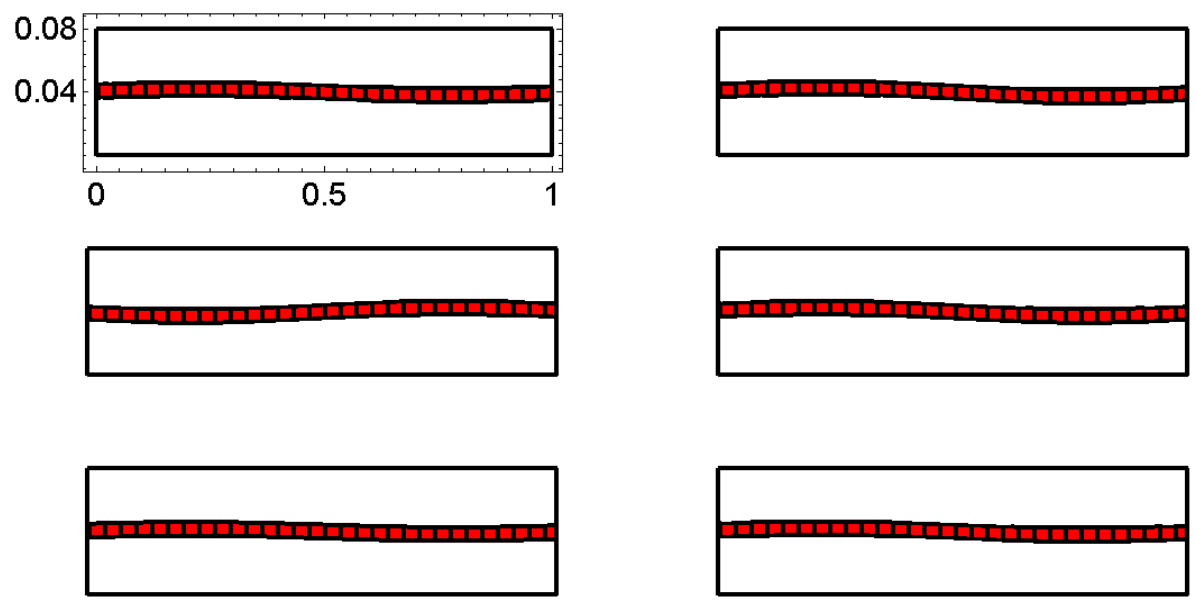

Figure 7: (colour online) Snapshots of the interface profile at $t=1 \mathrm{~s}, t=7.7 \mathrm{~s}$ for the first row from left to right, $t=12 \mathrm{~s}, t=16 \mathrm{~s}$ for the second row from left to right, and $t=24.5 \mathrm{~s}$, $t=32 \mathrm{~s}$ for the third row from left to right. The numerical solution is shown in solid line and the exact solution in dashed red line.
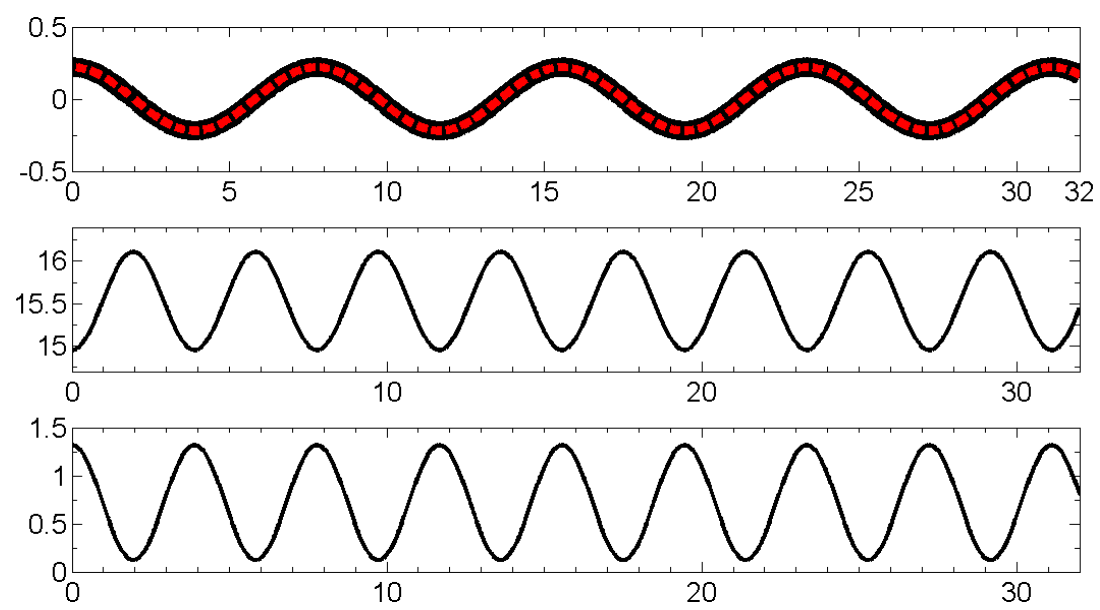

Figure 8: First row: numerical and exact solutions for the vessel motion up to $32 \mathrm{sec}$. The numerical solution is shown in solid line and the exact solution in dashed red line. The vertical axis is $q(m)$ and the horizontal axis is time. Second row: fluid energy versus time. Third row: vessel energy versus time.

at $x=.5 m$ versus time. The system constraints are preserved approximately with an error of order $\Delta t^{p}$ with $2<p<3$. Numerical errors are bounded over time.

The third simulation is for the $1: 1$ resonance with two non-Boussinesq fluids of densities $\rho_{1}=750 \mathrm{~kg} / \mathrm{m}^{3}$ and $\rho_{2}=1000 \mathrm{~kg} / \mathrm{m}^{3}$. The input parameters and the natural frequencies are

$$
\begin{aligned}
L & =1 \mathrm{~m}, \quad \nu=\nu_{1: 1}=167.24 \mathrm{~kg} / \mathrm{sec}^{2}, \quad h_{1}^{0}=.04 \mathrm{~m}, \quad h_{2}^{0}=.04 \mathrm{~m}, \quad \widehat{q}=.22 \mathrm{~m}, \\
\Delta x & =.01 \mathrm{~m}, \quad \Delta t=.001 \mathrm{sec}, \quad m_{f}^{1}=30 \mathrm{~kg}, \quad m_{f}^{2}=40 \mathrm{~kg}, \quad m_{v}=7 \mathrm{~kg}, \\
g & =9.81 \mathrm{~m} / \mathrm{sec}^{2}, \quad \mathrm{R}=52.89, \quad \mathrm{G}=522.10, \quad \omega^{\text {coupled }}=\omega_{1}^{\text {resonance }}=1.4876 \mathrm{rad} / \mathrm{sec}, \\
T^{\text {coupled }} & =4.2236 \mathrm{sec}, \quad \omega_{1}^{f}=.7438 \mathrm{rad} / \mathrm{sec}, \quad \omega_{f v}=1.4737 \mathrm{rad} / \mathrm{sec} .
\end{aligned}
$$




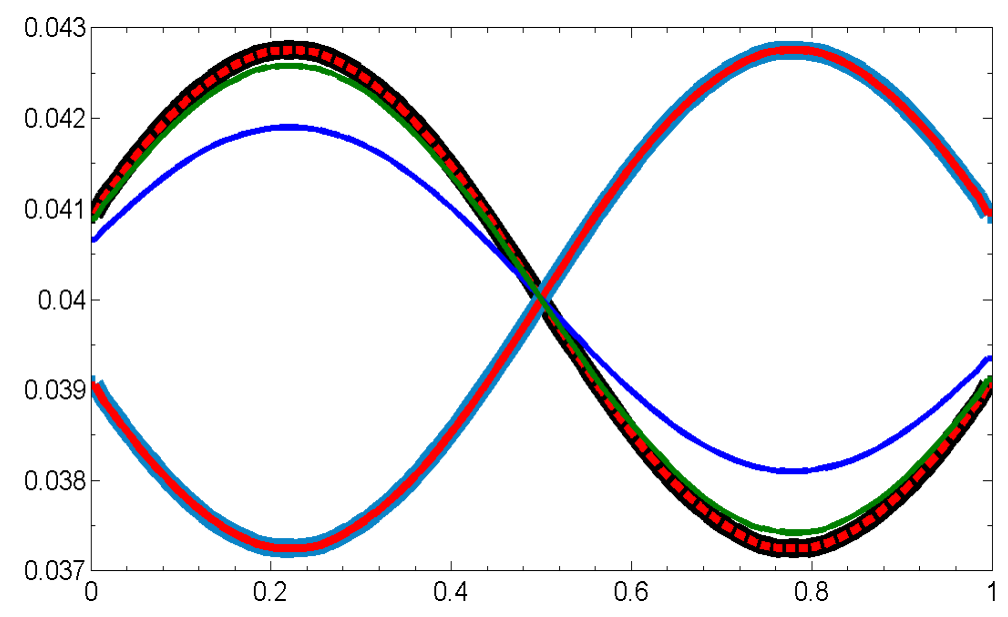

Figure 9: (colour online) Standing waves with one node for the root $s_{2}$ of the coupled oscillations with $\nu<\nu_{1: 1}$. Snapshots of the computed interface profile at $t=7.778 \mathrm{sec}$ in solid black line, at $t=4 \times 7.778 \mathrm{sec}$ in dashed red line, at $t=\frac{1}{2} \times 7.778 \mathrm{sec}$ in solid light blue line, at $t=\frac{1}{2} \times 7.778+3 \times 7.778 \mathrm{sec}$ in solid red line, at $t=1 \mathrm{sec}$ in dark blue line, and at $t=16 \mathrm{sec}$ in green line.

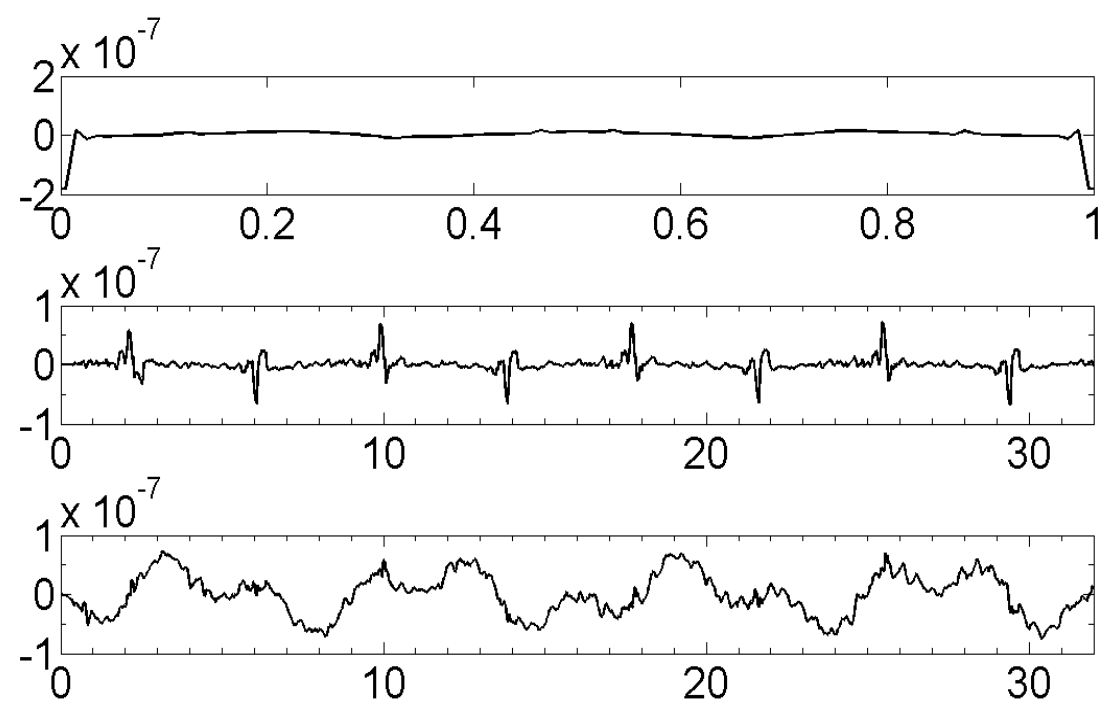

Figure 10: First row: $h_{1}^{0} \widetilde{u}_{1}+h_{2}^{0} \widetilde{u}_{2}$ at $t=32 \mathrm{sec}$. Second row: $\widetilde{h}_{1}+\widetilde{h}_{2}$ at $x=.5 m$ versus time. Third row: $h_{1}^{0} \widetilde{u}_{1}+h_{2}^{0} \widetilde{u}_{2}$ at $x=.5 m$ versus time.

Figure 11 shows the snapshots of the computed and exact wave profiles at different times. The numerical solutions are in very good agreement with the exact solutions. Numerical errors in preservation of the two constraints are shown in Figure 12. The first row in Figure 12 shows $h_{1}^{0} \widetilde{u}_{1}+h_{2}^{0} \widetilde{u}_{2}$ at $t=30 \mathrm{sec}$, the second row shows the conservation of the rigid-lid constraint $\widetilde{h}_{1}+\widetilde{h}_{2}=0$ at $x=.5 \mathrm{~m}$ versus time, and the third row shows $h_{1}^{0} \widetilde{u}_{1}+h_{2}^{0} \widetilde{u}_{2}=0$ at $x=.5 \mathrm{~m}$ versus time. The system constraints are preserved approximately with an error of order $\Delta t^{2}$, and numerical errors are bounded over time.

The first row in Figure 13 shows the numerical and exact solutions for the vessel displacement 

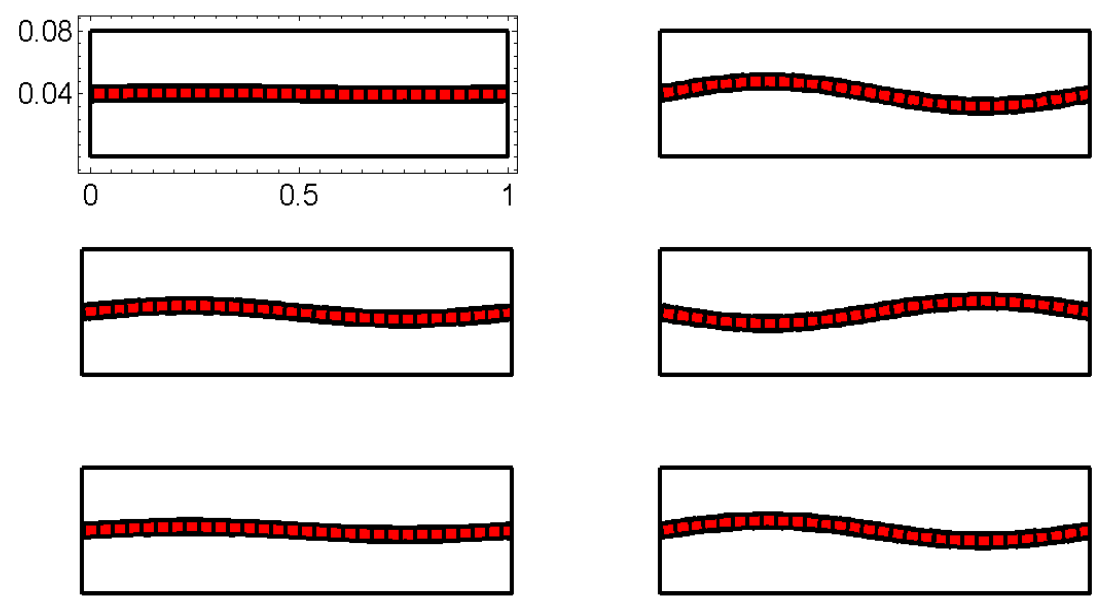

Figure 11: (colour online) Snapshots of the interface profile with two non-Boussinesq fluids at $t=1 \mathrm{~s}, t=4.2 \mathrm{~s}$ for the first row from left to right, $t=12 \mathrm{~s}, t=18.7 \mathrm{~s}$ for the second row from left to right, and $t=24.5 \mathrm{~s}, t=30 \mathrm{~s}$ for the third row from left to right. The horizontal and vertical axes are, respectively, $x(m)$ and $h_{2,1}(x, t)$. The numerical solution is shown in solid line and the exact solution in dashed red line.

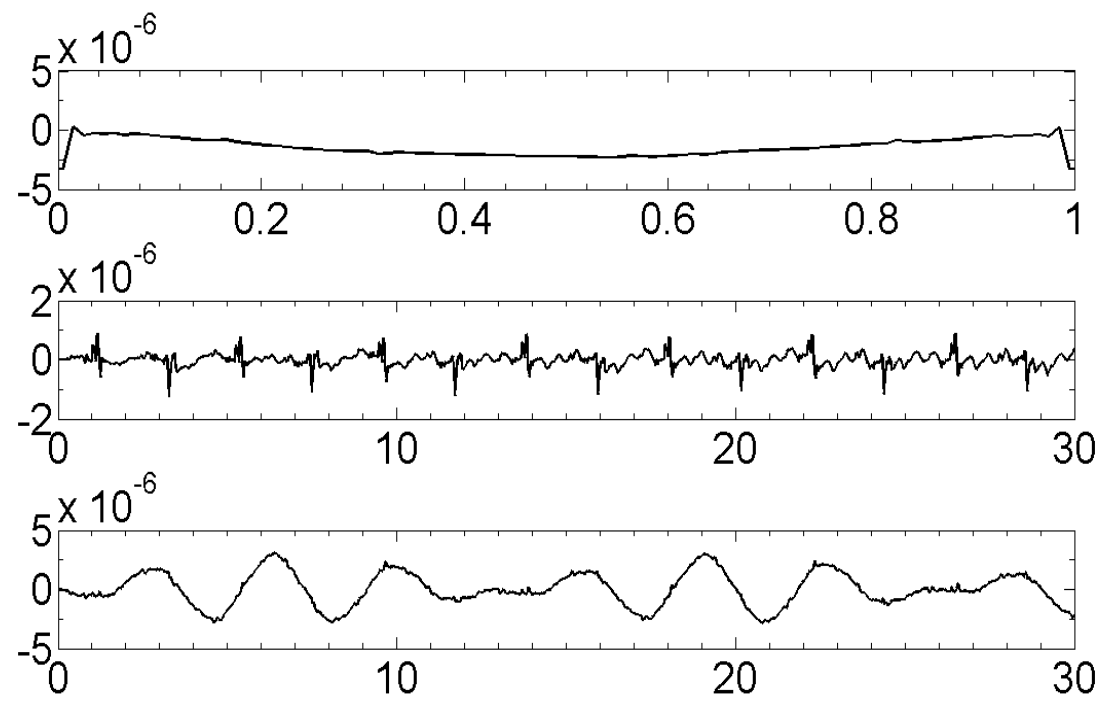

Figure 12: First row: $h_{1}^{0} \widetilde{u}_{1}+h_{2}^{0} \widetilde{u}_{2}$ at $t=30 \mathrm{sec}$. Second row: $\widetilde{h}_{1}+\widetilde{h}_{2}$ at $x=.5 \mathrm{~m}$ versus time. Third row: $h_{1}^{0} \widetilde{u}_{1}+h_{2}^{0} \widetilde{u}_{2}$ at $x=.5 m$ versus time.

versus time, the second row shows the energy of the fluid (6.2) versus time for the linear system, and the third row shows the energy of the vessel (6.1) versus time up to $30 \mathrm{sec}$. The partition of energy between the fluid and vessel motion is maintained.

The numerical solutions for the interface wave profile at $1: 1$ resonance at integer and half integer multiples of the coupled natural frequency (resonance frequency) $T^{\text {coupled }}=4.2236 \mathrm{sec}$ are depicted in Figure 14. The interface wave profile at $1: 1$ resonance resembles a standing wave with three nodes.

The next simulation is for the in phase oscillations between the fluids and the vessel motion 

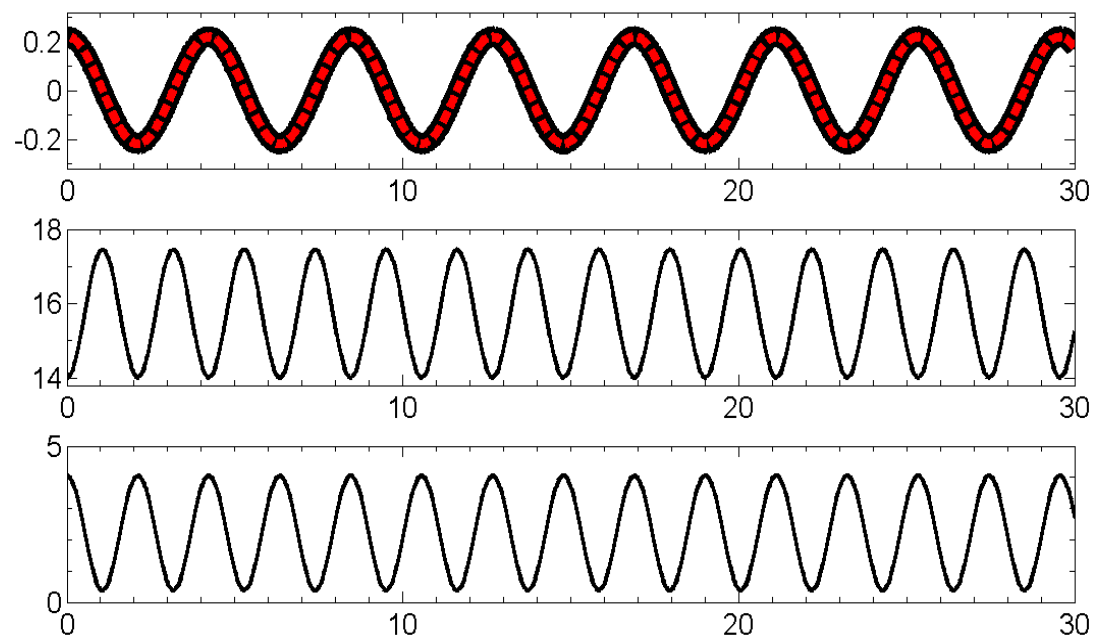

Figure 13: (colour online) First row: numerical and exact solutions for the vessel motion at internal $1: 1$ resonance for the coupled system with two non-Boussinesq fluids. The numerical solution is shown in solid line and the exact solution in dashed red line. The vertical axis is $q(m)$ and the horizontal axis is time. Second row: fluid energy versus time. Third row: vessel energy versus time.

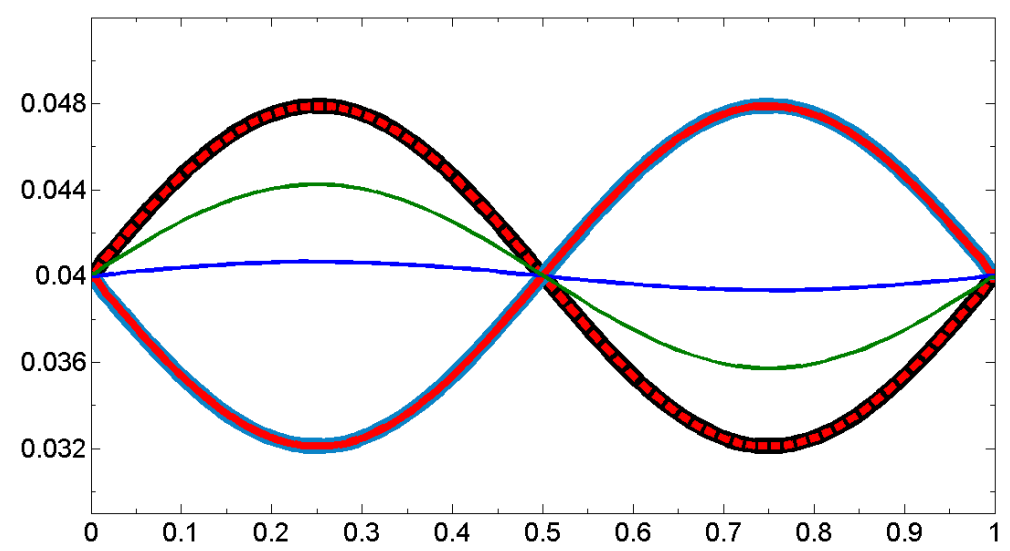

Figure 14: (colour online) Standing waves with three nodes at $1: 1$ resonance for two nonBoussinesq fluids. Snapshots of the numerical interface profile at $t=4.22 \mathrm{sec}$ in solid black line, at $t=4 \times 4.22 \mathrm{sec}$ in dashed red line, at $t=\frac{1}{2} \times 4.22 \mathrm{sec}$ in solid light blue line, at $t=\frac{1}{2} \times 4.22+3 \times 4.22 \mathrm{sec}$ in solid red line, at $t=1 \mathrm{sec}$ in dark blue line, and at $t=12 \mathrm{sec}$ in green line.

for the root $s_{1}$ of the coupled linear solutions in the non-Boussinesq regime. The two fluid densities are set as $\rho_{1}=400 \mathrm{~kg} / \mathrm{m}^{3}$ and $\rho_{2}=1000 \mathrm{~kg} / \mathrm{m}^{3}$. The other input parameters and 
natural frequencies are

$$
\begin{aligned}
L & =1 \mathrm{~m}, \quad \nu=226.11 \mathrm{~kg} / \mathrm{sec}^{2}, \quad \nu_{1: 1}=452.22 \mathrm{~kg} / \mathrm{sec}^{2}, \quad h_{1}^{0}=.04 \mathrm{~m}, \quad h_{2}^{0}=.04 \mathrm{~m}, \\
\widehat{q} & =.02 \mathrm{~m}, \quad \Delta x=.01 \mathrm{~m}, \quad \Delta t=.001 \mathrm{sec}, \quad m_{f}^{1}=16 \mathrm{~kg}, \\
m_{f}^{2} & =40 \mathrm{~kg}, \quad m_{v}=.4\left(m_{f}^{1}+m_{f}^{2}\right)=22.4 \mathrm{~kg}, \quad g=9.81 \mathrm{~m} / \mathrm{sec}^{2}, \quad \mathrm{R}=6.62, \\
\mathrm{G} & =32.67, \quad \omega_{1}^{\text {coupled }}=1.2207 \mathrm{rad} / \mathrm{sec}, \quad \omega_{1}^{\text {resonance }}=2.5766 \mathrm{rad} / \mathrm{sec}, \quad \mathrm{s}_{1}=1.48, \\
T^{\text {coupled }} & =5.1471 \mathrm{sec}, \quad \omega_{1}^{f}=1.2883 \mathrm{rad} / \mathrm{sec}, \quad \omega_{f v}=1.6982 \mathrm{rad} / \mathrm{sec} .
\end{aligned}
$$

Figure 15 shows the snapshots of the computed and exact interface profiles at different times. The numerical solutions are in very good agreement with the exact solutions.
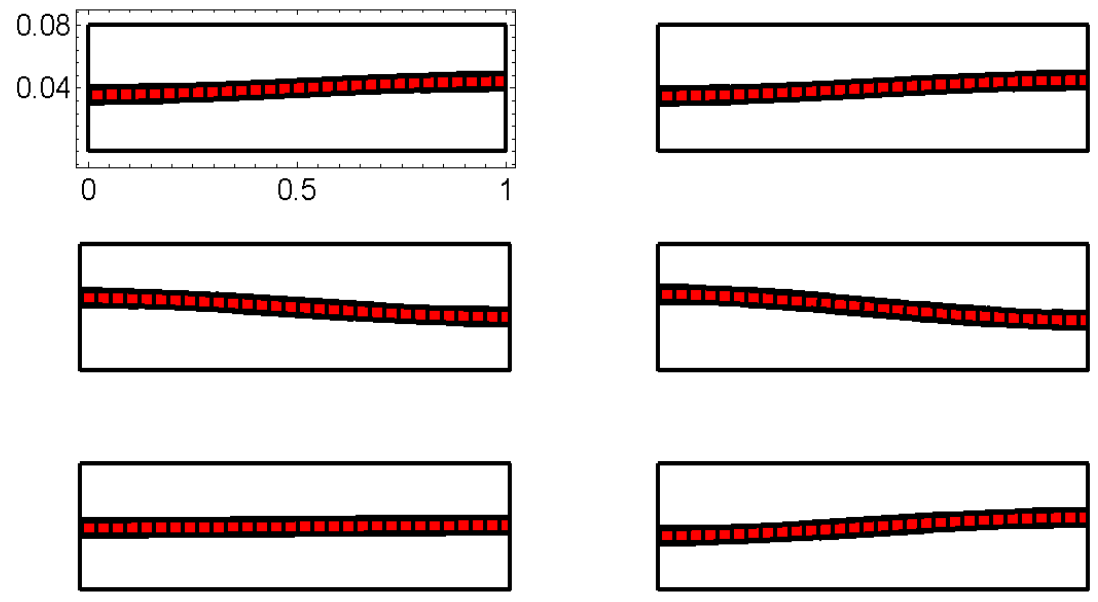

Figure 15: (colour online) Snapshots of the interface profile with two non-Boussinesq fluids of densities $\rho_{1}=400 \mathrm{~kg} / \mathrm{m}^{3}$ and $\rho_{2}=1000 \mathrm{~kg} / \mathrm{m}^{3}$ at $t=1 \mathrm{~s}, t=4.2 \mathrm{~s}$ for the first row from left to right, $t=12 \mathrm{~s}, t=18.7 \mathrm{~s}$ for the second row from left to right, and $t=24.5 \mathrm{~s}, t=30 \mathrm{~s}$ for the third row from left to right. The horizontal and vertical axes are, respectively, $x(m)$ and $h_{2,1}(x, t)$. The numerical solution is shown in solid line and the exact solution in dashed red line.

Numerical errors in preservation of the two constraints are shown in Figure 16. The first row in Figure 16 shows $h_{1}^{0} \widetilde{u}_{1}+h_{2}^{0} \widetilde{u}_{2}$ at $t=30 \mathrm{sec}$, the second row shows the conservation of the rigidlid constraint $\widetilde{h}_{1}+\widetilde{h}_{2}=0$ at $x=.5 m$ versus time, and the third row shows $h_{1}^{0} \widetilde{u}_{1}+h_{2}^{0} \widetilde{u}_{2}=0$ at $x=.5 \mathrm{~m}$ versus time. The system constraints in the non-Boussinesq limit are preserved approximately with an error of order $\Delta t^{2}$, and numerical errors are bounded over time.

The first row in Figure 17 shows the numerical and exact solutions for the vessel displacement versus time which are in very good agreement, the second row shows the energy of the fluid (6.2) versus time for the linear system, and the third row shows the energy of the vessel (6.1) versus time up to $30 \mathrm{sec}$. The partition of energy between the fluid and vessel motion is maintained.

The numerical solutions for the interface wave profile at integer and half integer multiples of the coupled natural frequency $T^{\text {coupled }}=5.147 \mathrm{sec}$ are depicted in Figure 18. The interface wave profile for the root $s_{1}$ of the coupled linear solutions resembles a standing wave with one node which oscillates in phase with the vessel motion. 


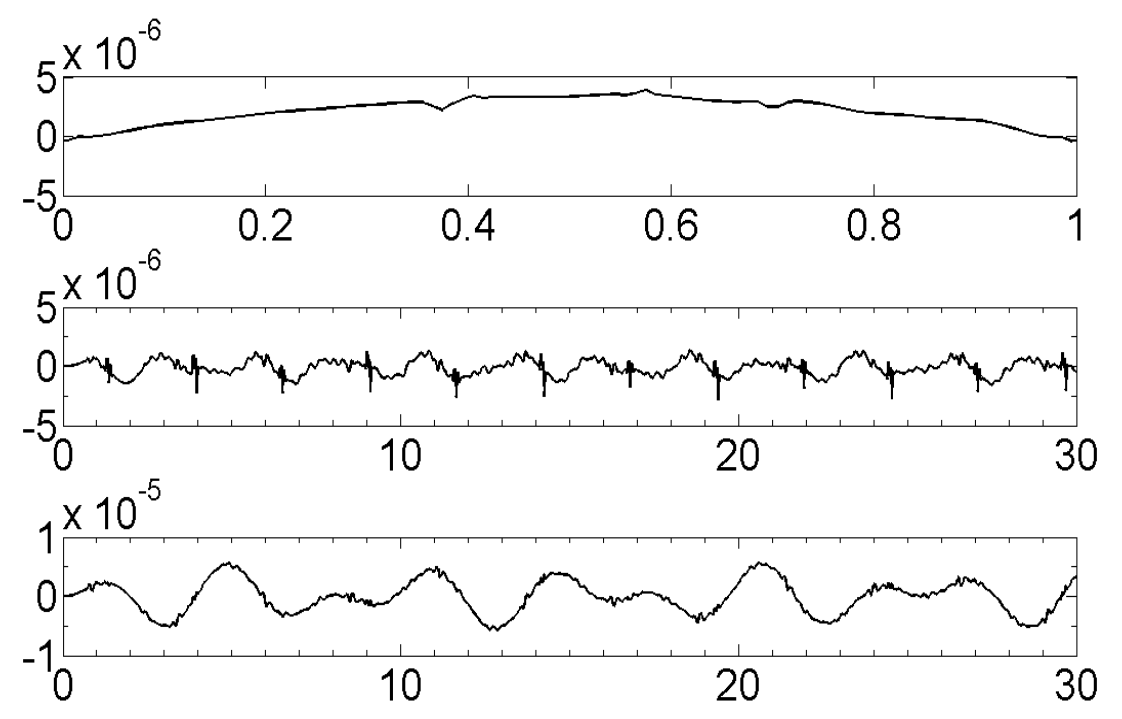

Figure 16: First row: $h_{1}^{0} \widetilde{u}_{1}+h_{2}^{0} \widetilde{u}_{2}$ at $t=30 \mathrm{sec}$. Second row: $\widetilde{h}_{1}+\widetilde{h}_{2}$ at $x=.5 m$ versus time. Third row: $h_{1}^{0} \widetilde{u}_{1}+h_{2}^{0} \widetilde{u}_{2}$ at $x=.5 m$ versus time.
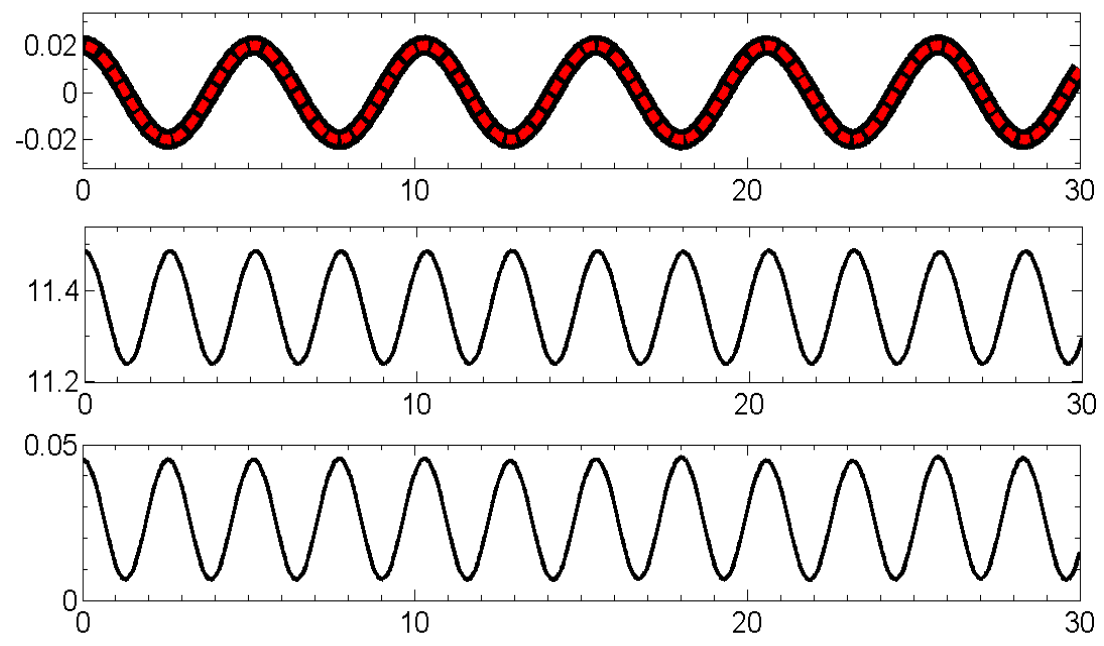

Figure 17: (colour online) First row: numerical and exact solutions for the vessel motion at root $s_{1}$ of the coupled system with two non-Boussinesq fluids of densities $\rho_{1}=400 \mathrm{~kg} / \mathrm{m}^{3}$ and $\rho_{2}=1000 \mathrm{~kg} / \mathrm{m}^{3}$. The numerical solution is shown in solid line and the exact solution in dashed red line. The vertical axis is $q(m)$ and the horizontal axis is time. Second row: fluid energy versus time. Third row: vessel energy versus time.

\subsubsection{Comparison of natural frequencies}

In this section the analytical results of $\$ 2.1$ and $\S 2.3$ on the natural frequencies of the coupled and uncoupled systems are verified for different spring constants and different density ratios. In table 1 the computed parameters of the coupled linear solutions are presented for a spring of constant $\nu=140 \mathrm{~kg} / \mathrm{sec}^{2}$ where $\mathrm{G}$ is much greater than one. In all cases $L, h_{1}^{0}, h_{2}^{0}$ and $\rho_{2}$ are held fixed while $\rho_{1}$ is changed with $m_{v}=.1\left(m_{f}^{1}+m_{f}^{2}\right)$. As is expected from the analytical 


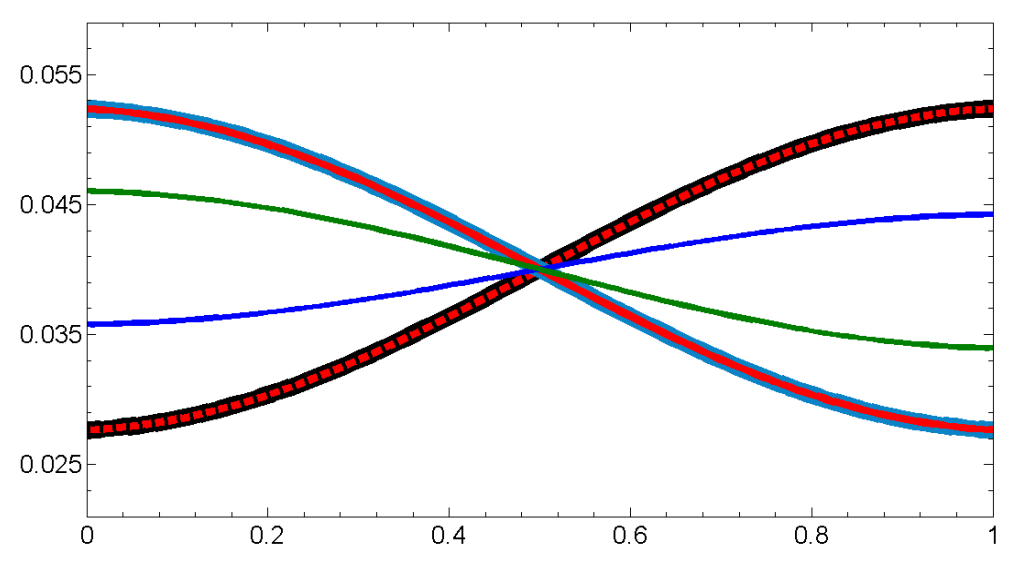

Figure 18: (colour online) In phase oscillations between the fluids and vessel motion for the root $s_{1}$ of the coupled linear solutions. Standing waves with one node for two non-Boussinesq fluids of densities $\rho_{1}=400 \mathrm{~kg} / \mathrm{m}^{3}$ and $\rho_{2}=1000 \mathrm{~kg} / \mathrm{m}^{3}$. Snapshots of the numerical interface profile at $t=5.147 \mathrm{sec}$ in solid black line, at $t=4 \times 5.147 \mathrm{sec}$ in dashed red line, at $t=$ $\frac{1}{2} \times 5.147 \mathrm{sec}$ in solid light blue line, at $t=\frac{1}{2} \times 5.147+3 \times 5.147 \mathrm{sec}$ in solid red line, at $t=1 \mathrm{sec}$ in dark blue line, and at $t=12 \mathrm{sec}$ in green line.

results, in all cases $\omega_{1}^{\text {coupled }}$ is less than $\omega_{1}^{f}$ and $\omega_{f v}$ and $\omega_{1}^{\text {resonance }}$ is twice $\omega_{1}^{f}$. Also for very large values of $\mathrm{G}$ where the fundamental coupled natural frequency $s_{1}$ becomes very close to $\pi / 2$, for the upper fluid densities $\rho_{2}=800$ and $\rho_{2}=900$, the coupled natural frequency $\omega_{1}^{\text {coupled }}$ goes to $\omega_{1}^{f}$. Our numerical solutions, for this particular spring constant, show that for such $s_{1}$ roots of $\mathrm{D}(s)=0$, the interface wave profile oscillates with $\omega_{1}^{f}$ and the vessel oscillates with $\omega_{f v}$ which does not appear to make physical sense. However, for the root $s_{2}$ of $\mathrm{D}(s)=0$, the coupled solutions oscillate with $\omega_{2}^{\text {coupled }}$. The interface wave profile for the $s_{2}$ roots resembles a standing wave with one or three nodes. In table 2 the computed parameters of the linear solutions are presented for a spring constant $\nu=40 \mathrm{~kg} / \mathrm{sec}^{2}$ for both Boussinesq and nonBoussinesq regimes. For the non-Boussinesq fluids $\mathrm{G}$ has small values. In all cases $L, h_{1}^{0}, h_{2}^{0}$ and $\rho_{2}$ are held fixed while $\rho_{1}$ is changed with $m_{v}=.1\left(m_{f}^{1}+m_{f}^{2}\right)$. As is expected, in all cases $\omega_{1}^{\text {coupled }}$ is less than $\omega_{1}^{f}$ and $\omega_{f v}$. In table 3 the natural frequencies of the coupled problem are reported at $1: 1$ resonance. The spring constant is set at $\nu_{1: 1}(2.25)$ with $(m=1)$ for different fluid densities. In all cases $L, h_{1}^{0}, h_{2}^{0}$ and $\rho_{2}$ are held fixed while $\rho_{1}$ is changed with $m_{v}=.1\left(m_{f}^{1}+m_{f}^{2}\right)$. As is shown in the table the important conclusion at $1: 1$ resonance is that the coupled natural frequency satisfies $\omega^{\text {coupled }}>\omega_{f v}>\omega_{1}^{f}$. Also since at $1: 1$ resonance $s \geq \pi$ it can be concluded that resonance only occurs at modes higher than the fundamental mode $s_{1}$. This agrees with the analytical argument made for the one layer problem in [16] and also for the baffled dynamic coupling problem in [44]. A comparison of the values of $G$ between the tables 3 and 2 shows that $G$ has much larger values at resonance. In the Boussinesq regime, the coupled natural frequency $\omega^{\text {coupled }}$ becomes very close to $\omega_{f v}$.

\subsection{Nonlinear simulations}

In this section we consider five simulations of the nonlinear system (3.2) and (5.6). Nonlinear simulations for other parameter sets can be found in [4]. The first simulation is a comparison 
Table 1: For all cases $\rho_{2}=1000 \mathrm{~kg} / \mathrm{m}^{3}, \mathrm{~m}_{f}^{2}=60 \mathrm{~kg}, \nu=140 \mathrm{~kg} / \mathrm{sec}^{2}, L=1 \mathrm{~m}, \mathrm{~h}_{1}^{0}=.05 \mathrm{~m}$ and $h_{2}^{0}=.06 \mathrm{~m}$. The dimension of the given parameters are as follows: $\rho_{1}$ in $\mathrm{kg} / \mathrm{m}^{3}, \mathrm{~m}_{f}^{1}$ and $m_{v}$ in $\mathrm{kg}, T^{\text {coupled }}$ in sec, $\omega_{1}^{\text {coupled }}, \omega_{1}^{f}, \omega_{f v}$ and $\omega_{1}^{\text {resonance }}$ in $\mathrm{rad} / \mathrm{sec}$, and $\nu_{1: 1}$ in $\mathrm{kg} / \mathrm{sec}^{2}$.

\begin{tabular}{cccccccccccc}
\hline$\rho_{1}$ & $m_{f}^{1}$ & $m_{v}$ & $s_{1}$ & $\mathrm{R}$ & $\mathrm{G}$ & $T^{\text {coupled }}$ & $\omega_{1}^{\text {coupled }}$ & $\omega_{1}^{f}$ & $\omega_{f v}$ & $\omega_{1}^{\text {resonance }}$ & $\nu_{1: 1}$ \\
\hline 500 & 25 & 8.5 & 1.3097 & 8.97 & 20.29 & 5.5927 & 1.1234 & 1.3473 & 1.2236 & 2.6947 & 610.87 \\
\hline 600 & 30 & 9 & 1.4411 & 16.73 & 45.81 & 5.8921 & 1.0663 & 1.1623 & 1.1891 & 2.3246 & 504.82 \\
\hline 700 & 35 & 9.5 & 1.5348 & 34.60 & 124.27 & 6.6070 & .9509 & .9732 & 1.1574 & 1.9464 & 384.79 \\
\hline 800 & 40 & 10 & 1.5647 & 88.83 & 475.90 & 8.1923 & .7669 & .7699 & 1.1281 & 1.5398 & 257.92 \\
\multicolumn{7}{c}{$s_{2}=2.3206$} & & & 5.5239 & $\omega_{2}=1.1374$ \\
\hline 900 & 45 & 10.5 & 1.5694 & 399.40 & 4287.6 & 11.8997 & .5280 & .5284 & 1.1009 & 1.0569 & 128.71 \\
& \multicolumn{7}{c}{$s_{2}=3.2763$} & & & 5.7001 & $\omega_{2}=1.1022$ \\
\end{tabular}

Table 2: For all cases $\rho_{2}=1000 \mathrm{~kg} / \mathrm{m}^{3}, \mathrm{~m}_{f}^{2}=60 \mathrm{~kg}, \nu=40 \mathrm{~kg} / \mathrm{sec}^{2}, L=1 \mathrm{~m}, \mathrm{~h}_{1}^{0}=.06 \mathrm{~m}$ and $h_{2}^{0}=.06 \mathrm{~m}$. The dimension of the given parameters are as follows: $\rho_{1}$ in $\mathrm{kg} / \mathrm{m}^{3}, \mathrm{~m}_{f}^{1}$ and $m_{v}$ in $\mathrm{kg}, T^{\text {coupled }}$ in sec, $\omega_{1}^{\text {coupled }}, \omega_{1}^{f}, \omega_{f v}$ and $\omega_{1}^{\text {resonance }}$ in $\mathrm{rad} / \mathrm{sec}$, and $\nu_{1: 1}$ in $\mathrm{kg} / \mathrm{sec}^{2}$.

\begin{tabular}{cccccccccccc}
\hline$\rho_{1}$ & $m_{f}^{1}$ & $m_{v}$ & $s_{1}$ & $\mathrm{R}$ & $\mathrm{G}$ & $T^{\text {coupled }}$ & $\omega_{1}^{\text {coupled }}$ & $\omega_{1}^{f}$ & $\omega_{f v}$ & $\omega_{1}^{\text {resonance }}$ & $\nu_{1: 1}$ \\
\hline 200 & 12 & 7.2 & .5543 & 1.475 & .796 & 9.0474 & .6944 & 1.9679 & .7106 & 3.9359 & 731.19 \\
\hline 300 & 18 & 7.8 & .5956 & 2.793 & 1.395 & 9.3679 & .6707 & 1.7686 & .6827 & 3.5372 & 790.58 \\
\hline 500 & 30 & 9 & .710 & 8.90 & 5.096 & 9.9893 & .6289 & 1.3915 & .6356 & 2.7831 & 689.36 \\
\hline 600 & 36 & 9.6 & .7958 & 16.599 & 11.326 & 10.2907 & .6105 & 1.2051 & .6154 & 2.4102 & 578.60 \\
\hline 750 & 45 & 10.5 & 1.0093 & 52.899 & 55.498 & 10.7336 & .5853 & .9109 & .5884 & 1.8219 & 376.29 \\
\hline 900 & 54 & 11.4 & 1.5491 & 396.10 & 1022.19 & 11.5217 & .5453 & .5529 & .5647 & 1.1058 & 152.97 \\
\hline
\end{tabular}

Table 3: Natural frequencies at $1: 1$ resonance. For all cases $\rho_{2}=1000 \mathrm{~kg} / \mathrm{m}^{3}, \mathrm{~m}_{f}^{2}=60 \mathrm{~kg}$, $L=1 \mathrm{~m}, h_{1}^{0}=.06 \mathrm{~m}, h_{2}^{0}=.06 \mathrm{~m}, \nu=\nu_{1: 1}, \omega^{\text {coupled }}=\omega_{1}^{\text {resonance }}$ and $s=s_{1}^{\text {resonance }}=\pi$. The dimension of the given parameters are as follows: $\rho_{1}$ in $\mathrm{kg} / \mathrm{m}^{3}, \mathrm{~m}_{f}^{1}$ and $m_{v}$ in $\mathrm{kg}, T^{\text {coupled }}$ in sec, $\omega^{\text {coupled }}, \omega_{1}^{f}$ and $\omega_{f v}$ in $\mathrm{rad} / \mathrm{sec}$, and $\nu_{1: 1}$ in $\mathrm{kg} / \mathrm{sec}^{2}$.

\begin{tabular}{cccccccccc}
\hline$\rho_{1}$ & $m_{f}^{1}$ & $m_{v}$ & $\mathrm{R}$ & $\mathrm{G}$ & $T^{\text {coupled }}$ & $\omega^{\text {coupled }}=\omega_{1}^{\text {resonance }}$ & $\omega_{1}^{f}$ & $\omega_{f v}$ & $\nu=\nu_{1: 1}$ \\
\hline 200 & 12 & 7.2 & 1.475 & 14.557 & 1.5963 & 3.9359 & 1.9679 & 3.0384 & 731.19 \\
\hline 300 & 18 & 7.8 & 2.793 & 27.574 & 1.7762 & 3.5372 & 1.7686 & 3.0354 & 790.58 \\
\hline 500 & 30 & 9 & 8.90 & 87.839 & 2.2576 & 2.7831 & 1.3915 & 2.6388 & 689.36 \\
\hline 600 & 36 & 9.6 & 16.599 & 163.835 & 2.6068 & 2.4102 & 1.2051 & 2.3407 & 578.60 \\
\hline 750 & 45 & 10.5 & 52.899 & 522.102 & 3.4485 & 1.8219 & .9109 & 1.8049 & 376.29 \\
\hline 900 & 54 & 11.4 & 396.10 & 3909.35 & 5.6815 & 1.1058 & .5529 & 1.1044 & 152.97 \\
\hline
\end{tabular}


for the vessel motion between the linear coupled equations and the nonlinear coupled equations but with $\nu_{2}=0$ in the vessel equation, i.e. nonlinear fluids and a linear vessel. Initial conditions are the linear solutions at $t=0 \mathrm{sec}$, and the boundary conditions are the no-flow boundary conditions. Set $m=1$ in (2.24) and take the input parameters as

$$
\begin{aligned}
\rho_{1} & =950 \mathrm{~kg} / \mathrm{m}^{3}, \quad \rho_{2}=1000 \mathrm{~kg} / \mathrm{m}^{3}, \quad L=1 \mathrm{~m}, \quad \nu_{1}=.5 \nu_{1: 1}=26.6098 \mathrm{~kg} / \mathrm{sec}^{2}, \\
h_{1}^{0} & =.05 \mathrm{~m}, \quad h_{2}^{0}=.05 \mathrm{~m}, \quad \widehat{q}=.1 \mathrm{~m}, \quad \Delta x=.01 \mathrm{~m}, \quad \Delta t=.001 \mathrm{sec}, \\
g & =9.81 \mathrm{~m} / \mathrm{sec}^{2}, \quad m_{v}=.1\left(m_{f}^{1}+m_{f}^{2}\right)=9.75 \mathrm{~kg}, \quad \mathrm{R}=1672.1, \quad \mathrm{G}=8251.4, \\
s_{2} & =2.22, \quad \omega_{2}^{\text {coupled }}=.4983 \mathrm{rad} / \mathrm{sec}, \quad T^{\text {coupled }}=12.6081 \mathrm{sec}, \quad \omega_{f v}=.4981 \mathrm{rad} / \mathrm{sec}, \\
\omega_{1}^{f} & =.3523 \mathrm{rad} / \mathrm{sec}, \quad m_{f}^{1}=47.5 \mathrm{~kg}, \quad m_{f}^{2}=50 \mathrm{~kg} .
\end{aligned}
$$

For the vessel displacement, the numerical solution for the nonlinear coupled system is compared with the exact analytical solution for the linear coupled problem in Figure 19. The first row in Figure 19 shows the numerical and the exact linear solutions for the vessel displacement versus time. This plot shows that the numerical solution for the vessel motion, with $\nu_{2}=0$ and with interior two nonlinear fluids sloshing, is very close to the linear coupled solution. The second row shows the energy of the fluid (6.2) versus time for the nonlinear system, and the third row shows the energy of the vessel versus time up to $50 \mathrm{sec}$. The vessel energy is

$$
E_{v}=\frac{1}{2} m_{v} \dot{q}^{2}+\frac{1}{2} \nu_{1} q^{2}-\frac{1}{4} \nu_{2} q^{4}
$$

The snapshots of the interface wave profile for the nonlinear coupled system are shown in
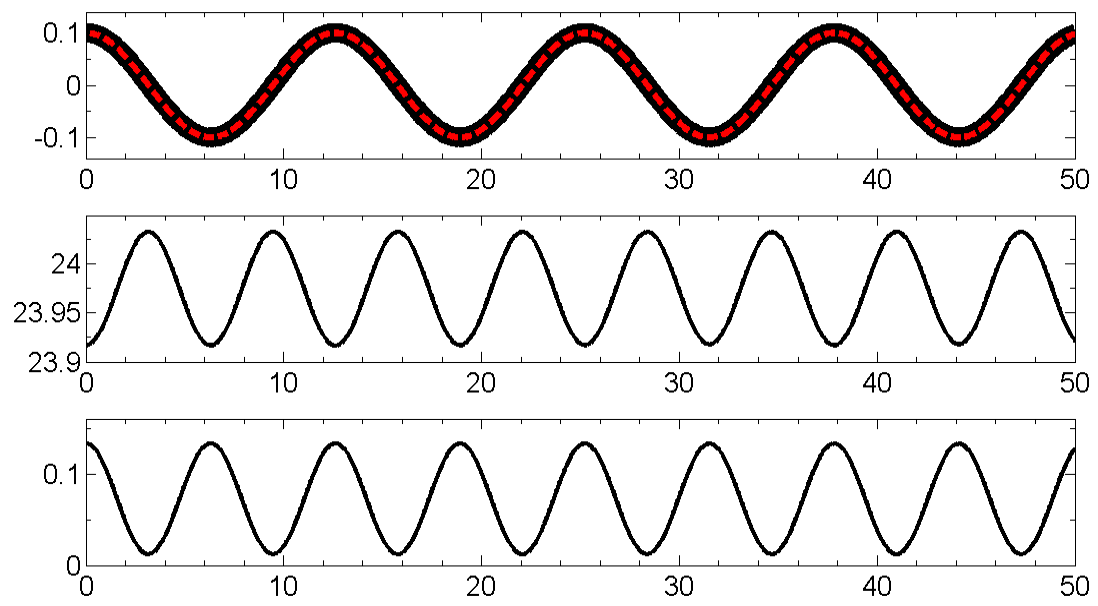

Figure 19: (colour online) First row: computed numerical solution for the vessel motion for the nonlinear coupled problem with $\nu_{2}=0$ against the exact solution for the linear coupled system. The numerical solution is shown in solid line and the exact solution in dashed red line. The vertical axis is $q(m)$ and the horizontal axis is time. Second row: nonlinear fluid energy versus time. Third row: vessel energy versus time.

Figure 20 against the exact linear solutions up to $50 \mathrm{sec}$. The nonlinear numerical and the exact linear solutions are very close for the coupled system with two fluids in the Boussinesq limit. Numerical errors in preservation of the two constraints are shown in Figure 21. The first row 

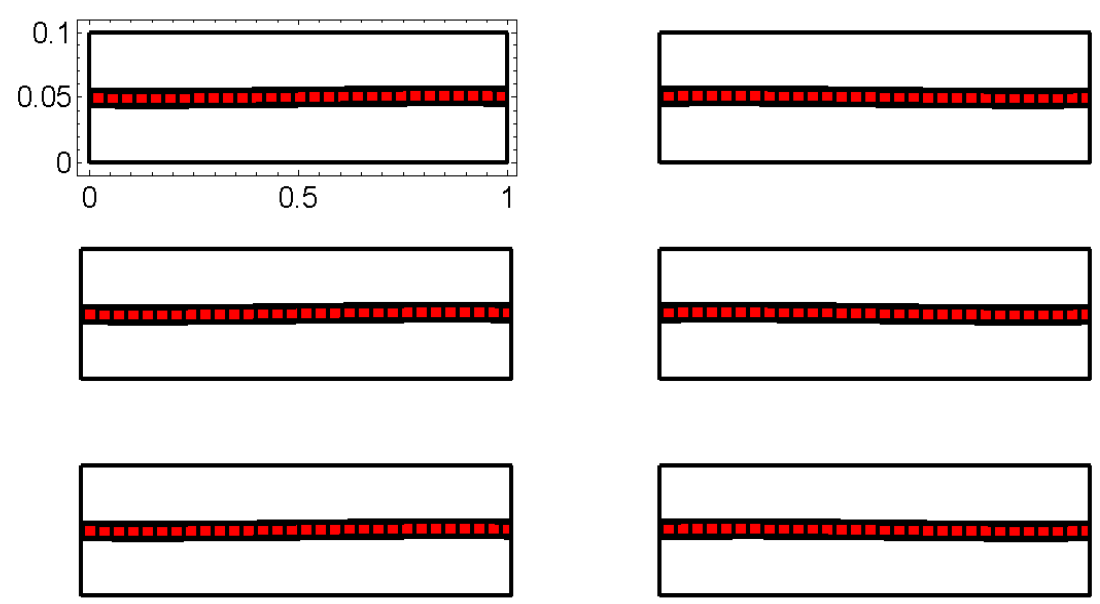

Figure 20: Snapshots of the nonlinear interface wave profile (black line) against the exact linear solutions (dashed red line) at $t=6.304 \mathrm{~s}, t=12.608 \mathrm{~s}$ for the first row from left to right, $t=19 \mathrm{~s}, t=25.216 \mathrm{~s}$ for the second row from left to right, and $t=31.52 \mathrm{~s}, t=50 \mathrm{~s}$ for the third row from left to right.

in Figure 21 shows $h_{1} u_{1}+h_{2} u_{2}$ at $t=50 \mathrm{sec}$, the second row shows the error of the rigid-lid constraint $h_{1}+h_{2}-d$ at $x=.5 \mathrm{~m}$ versus time, and the third row shows the error of the volume flux $h_{1} u_{1}+h_{2} u_{2}$ at $x=.50 \mathrm{~m}$ versus time. The system constraints are preserved approximately with an error of order $\Delta t^{p}$ with $1<p<2$.

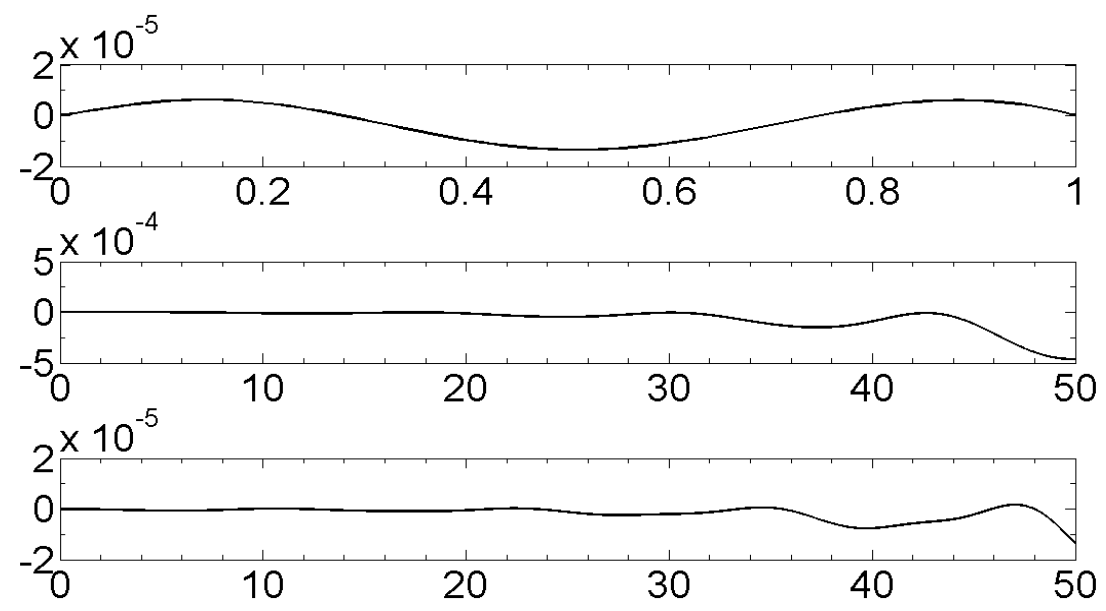

Figure 21: First row: $h_{1} u_{1}+h_{2} u_{2}$ at $t=50 \mathrm{sec}$. Second row: $h_{1}+h_{2}-d$ at $x=.50 \mathrm{~m}$ versus time. Third row: $h_{1} u_{1}+h_{2} u_{2}$ at $x=.50 \mathrm{~m}$ versus time.

The next simulation is a comparison for the vessel motion between the linear coupled equations and the nonlinear coupled equations with $\nu_{2}=0$ at internal $1: 1$ resonance. Set $m=1$ in 
(2.24) and take the input parameters as

$$
\begin{aligned}
\rho_{1} & =950 \mathrm{~kg} / \mathrm{m}^{3}, \quad \rho_{2}=1000 \mathrm{~kg} / \mathrm{m}^{3}, \quad L=1 \mathrm{~m}, \quad \nu_{1}=\nu_{1: 1}=64.1622 \mathrm{~kg} / \mathrm{sec}^{2}, \\
h_{1}^{0} & =.05 \mathrm{~m}, \quad h_{2}^{0}=.06 \mathrm{~m}, \quad \widehat{q}=.06 \mathrm{~m}, \quad \Delta x=.01 \mathrm{~m}, \quad \Delta t=.001 \mathrm{sec}, \\
g & =9.81 \mathrm{~m} / \mathrm{sec}^{2}, \quad m_{v}=.1\left(m_{f}^{1}+m_{f}^{2}\right)=10.75 \mathrm{~kg} .
\end{aligned}
$$

For the vessel displacement, the numerical solution for the nonlinear coupled system is compared with the exact analytical solution for the linear coupled problem in Figure 22. The first row in Figure 22 shows the numerical and exact solutions for the vessel displacement versus time, the second row shows the energy of the fluid (6.2) versus time for the nonlinear system, and the third row shows the energy of the vessel versus time up to $30 \mathrm{sec}$. The numerical solution is very close to the exact linear solution when $\nu_{2}=0$. The partition of energy between the fluid and vessel motion is maintained. The snapshots of the interface wave profile for the nonlinear
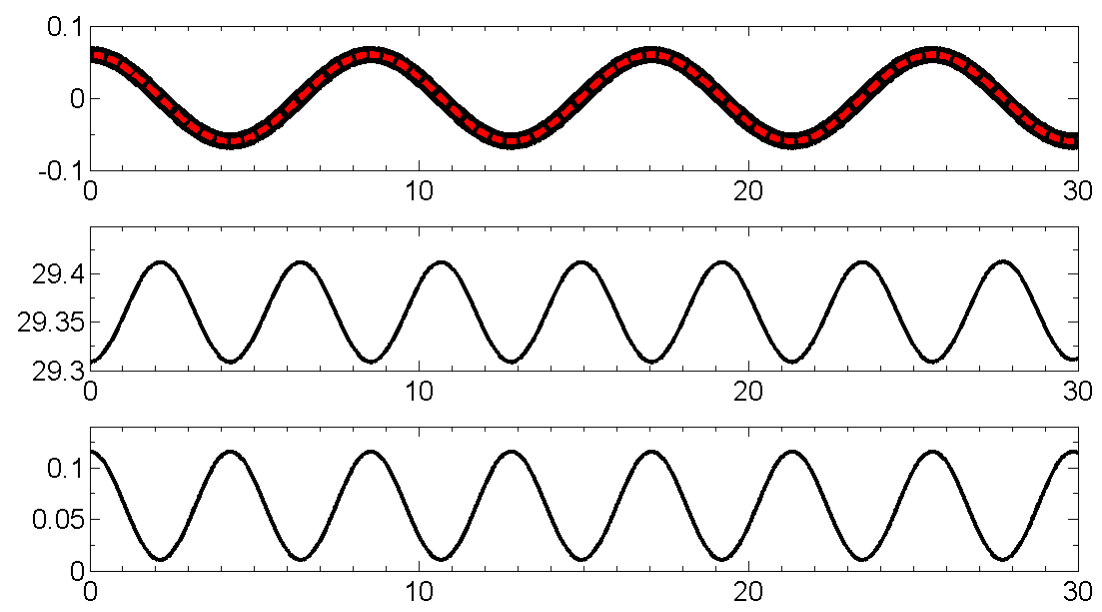

Figure 22: (colour online) First row: computed numerical solution for the vessel motion for the nonlinear coupled problem with $\nu_{2}=0$ at internal $1: 1$ resonance against the exact solution for the linear coupled system. The numerical solution is shown in solid line and the exact solution in dashed red line. The vertical axis is $q(m)$ and the horizontal axis is time. Second row: nonlinear fluid energy versus time. Third row: vessel energy versus time.

coupled system at $1: 1$ resonance are shown against the exact linear solutions in Figure 23 up to $30 \mathrm{sec}$. The interface profile resembles a standing wave with three nodes similar to the case with linear fluid equations.

The next simulation is for the full nonlinear coupled system with $\nu_{1} \neq 0$ and $\nu_{2} \neq 0$. Take the fluids to be quiescent

$$
u_{1}(x, 0)=u_{2}(x, 0)=0,
$$

and take the following initial conditions for the vessel position and velocity, and the interface wave profile at $t=0 \mathrm{sec}$,

$$
\begin{aligned}
h_{2}(x, 0) & =h_{2}^{0}+\frac{\omega^{2}}{g \alpha}[\sin (\alpha x)-\tan (s) \cos (\alpha x)] \widehat{q}, \\
q(0) & =\widehat{q} \quad \text { and } \quad \dot{q}(0)=0,
\end{aligned}
$$



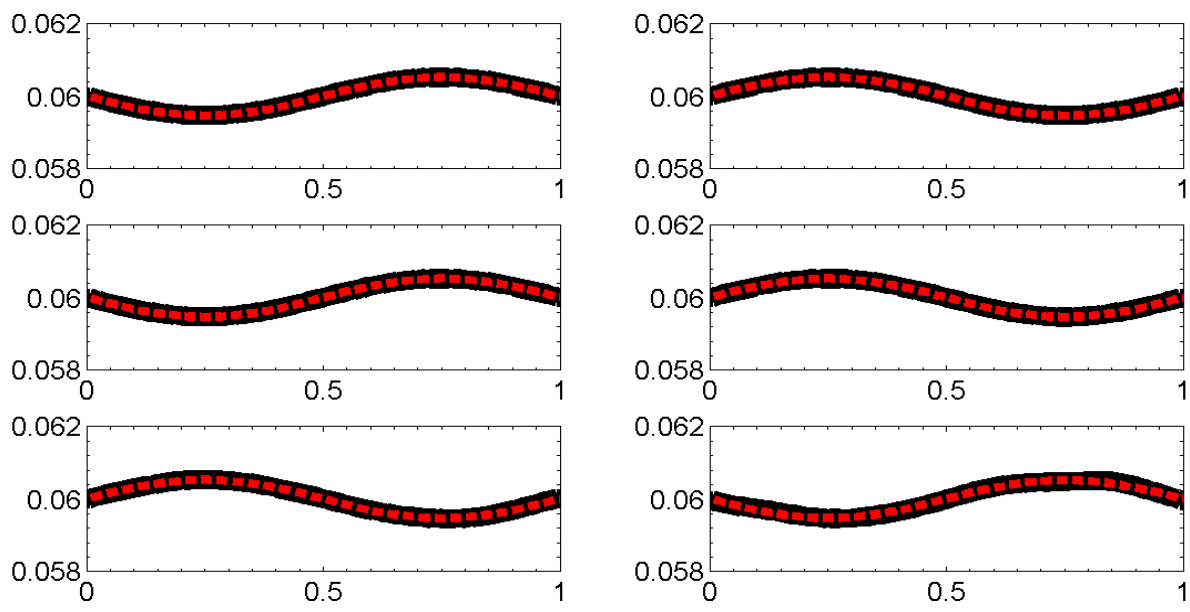

Figure 23: (colour online) Snapshots of the nonlinear interface wave profile against the exact linear solution at internal $1: 1$ resonance and in the Boussinesq limit at $t=4.264 \mathrm{~s}, t=8.528 \mathrm{~s}$ for the first row from left to right, $t=12.792 \mathrm{~s}, t=17.056 \mathrm{~s}$ for the second row from left to right, and $t=25.584 \mathrm{~s}, t=30 \mathrm{~s}$ for the third row from left to right. The numerical solution is shown in solid line and the exact solution in dashed red line.

with the input parameters

$$
\begin{aligned}
\rho_{1} & =900 \mathrm{~kg} / \mathrm{m}^{3}, \quad \rho_{2}=1000 \mathrm{~kg} / \mathrm{m}^{3}, \quad \widehat{q}=.06 \mathrm{~m}, \quad L=1 \mathrm{~m}, \\
\nu_{1} & =.25 \nu_{1: 1}=38.24 \mathrm{~kg} / \mathrm{sec}^{2}, \quad \nu_{2}=-.5 \nu_{1: 1}=-76.48 \mathrm{~kg} /\left(\mathrm{sec}^{2} \mathrm{~m}^{2}\right), \\
h_{1}^{0} & =.06 \mathrm{~m}, \quad h_{2}^{0}=.06 \mathrm{~m}, \quad \omega_{1}^{\text {coupled }}=.5405 \mathrm{rad} / \mathrm{sec}, \\
T^{\text {coupled }} & =11.624 \mathrm{sec}, \quad \Delta x=.01 \mathrm{~m}, \quad \Delta t=.001 \mathrm{sec}, \quad \mathrm{g}=9.81 \mathrm{~m} / \mathrm{sec}^{2}, \\
m_{v} & =.1\left(m_{f}^{1}+m_{f}^{2}\right)=11.4 \mathrm{~kg} .
\end{aligned}
$$

The vessel displacement for the full nonlinear coupled system is plotted in Figure 24. The first row in Figure 24 shows the computed numerical solution for the vessel displacement against the exact linear solution versus time. The nonlinear numerical and exact solutions are very close to each other. The second row shows the energy of the fluid (6.2) versus time for the nonlinear system, and the third row shows the energy of the vessel (6.3) versus time up to $50 \mathrm{sec}$. The snapshots of the computed interface wave profile against the exact linear solution are shown in Figure 25 up to $50 \mathrm{sec}$. The error in $h_{1} u_{1}+h_{2} u_{2}$ for $5 \times 10^{4}$ time steps is of order $10^{-5}$ and the error in $h_{1}+h_{2}-d$ is of order $10^{-4}$.

For the next simulation set $r=.9$ and take the fluids to be quiescent (6.4). Take the initial condition for the interface wave profile at $t=0 \mathrm{sec}$ as in (6.5) with the input parameters

$$
\begin{aligned}
\rho_{1} & =900 \mathrm{~kg} / \mathrm{m}^{3}, \quad \rho_{2}=1000 \mathrm{~kg} / \mathrm{m}^{3}, \quad \widehat{q}=.3 \mathrm{~m}, \quad L=1 \mathrm{~m}, \\
\nu_{1} & =.5 \nu_{1: 1}=76.48 \mathrm{~kg} / \mathrm{sec}^{2}, \quad \nu_{2}=2 \nu_{1: 1}=305.95 \mathrm{~kg} /\left(\mathrm{sec}^{2} \mathrm{~m}^{2}\right), \\
h_{1}^{0} & =.06 \mathrm{~m}, \quad h_{2}^{0}=.06 \mathrm{~m}, \quad \Delta x=.01 \mathrm{~m}, \quad \Delta t=.001 \mathrm{sec}, \\
g & =9.81 \mathrm{~m} / \mathrm{sec}^{2}, \quad m_{v}=.1\left(m_{f}^{1}+m_{f}^{2}\right)=11.4 \mathrm{~kg} .
\end{aligned}
$$

And take the initial conditions for the vessel position and velocity at $t=0 \mathrm{sec}$ as in (6.5) but with $\widehat{q}=-.02 \mathrm{~m}$. The vessel displacement for the full nonlinear coupled system is plotted in 

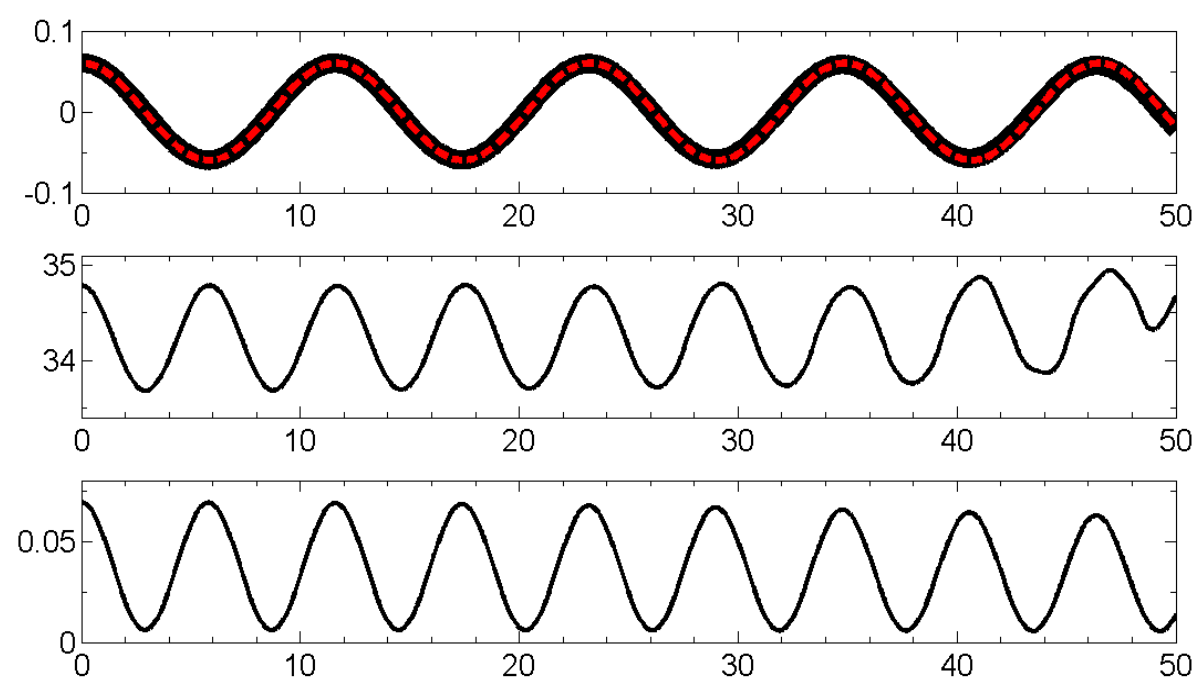

Figure 24: First row: computed numerical solution (black line) for the vessel motion for the nonlinear coupled system with $\nu_{1} \neq 0$ and $\nu_{2} \neq 0$ against the exact linear solution (dashed red line). The vertical axis is $q(m)$ and the horizontal axis is time. Second row: nonlinear fluid energy versus time. Third row: vessel energy versus time.
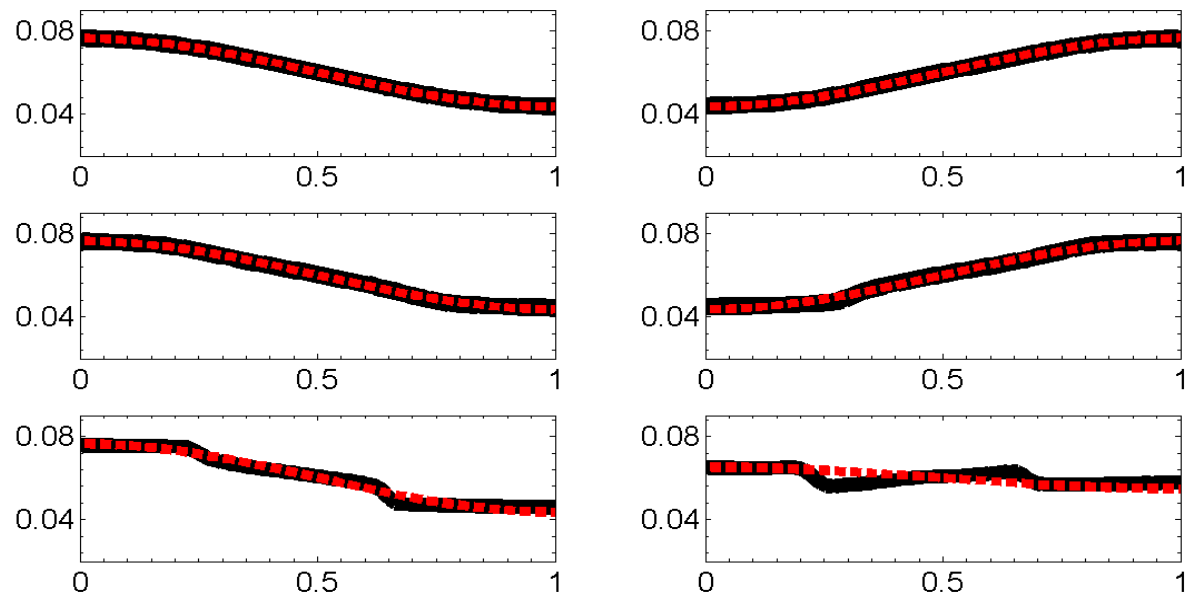

Figure 25: Snapshots of the nonlinear interface wave profile (black line) against the exact linear solution (dashed red line) at $t=5.812 \mathrm{~s}, t=11.624 \mathrm{~s}$ for the first row from left to right, $t=17.436 \mathrm{~s}, t=23.248 \mathrm{~s}$ for the second row from left to right, and $t=40.684 \mathrm{~s}, t=50 \mathrm{~s}$ for the third row from left to right.

Figure 26. The first row in Figure 26 shows the computed numerical solution for the vessel displacement versus time, the second row shows the energy of the fluid (6.2) versus time for the nonlinear system, and the third row shows the energy of the vessel (6.3) versus time up to $40 \mathrm{sec}$. The partition of energy between the fluid and vessel motion is maintained. The snapshots of the interface wave profile are shown in Figure 27 up to $40 \mathrm{sec}$. Numerical errors in preservation of the two constraints are shown in Figure 28. The first row in Figure 28 shows $h_{1} u_{1}+h_{2} u_{2}$ at $t=40 \mathrm{sec}$, the second row in Figure 28 shows the conservation of the rigid-lid constraint $h_{1}+h_{2}-d=0$ at $x=.5 \mathrm{~m}$ versus time, and the third row shows $h_{1} u_{1}+h_{2} u_{2}=0$ at $x=.5 \mathrm{~m}$ 

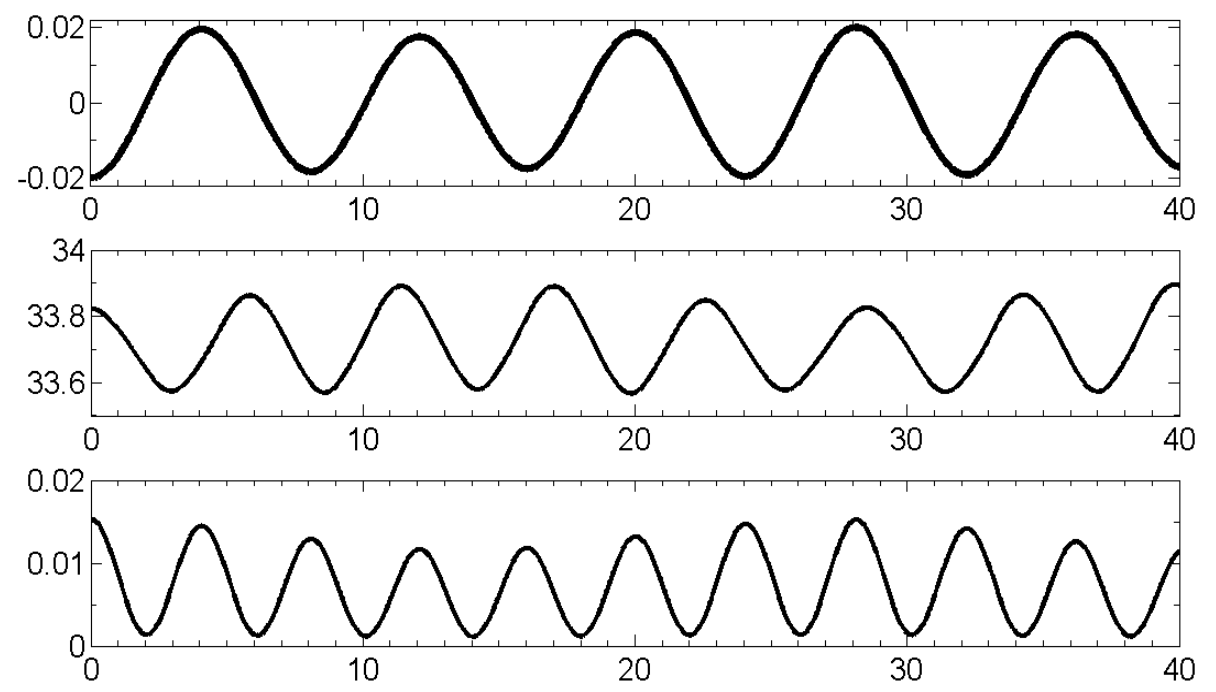

Figure 26: First row: computed numerical solution for the vessel motion for the nonlinear coupled system with $\nu_{1} \neq 0$ and $\nu_{2} \neq 0$. The vertical axis is $q(m)$ and the horizontal axis is time. Second row: nonlinear fluid energy versus time. Third row: vessel energy versus time.
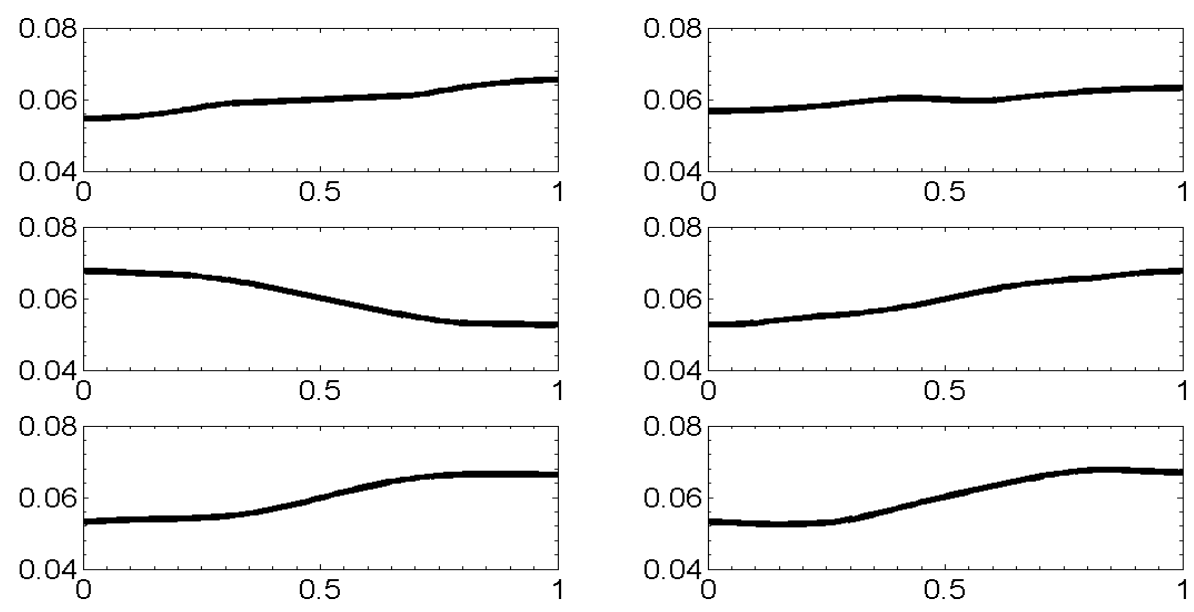

Figure 27: Snapshots of the nonlinear interface wave profile at $t=4.014 \mathrm{~s}, t=8.028 \mathrm{~s}$ for the first row from left to right, $t=12.042 \mathrm{~s}, t=16.056 \mathrm{~s}$ for the second row from left to right, and $t=28.098 \mathrm{~s}, t=40 \mathrm{~s}$ for the third row from left to right.

versus time. The system constraints are preserved approximately with an error of order $\Delta t^{p}$ with $1<p<2$.

The next simulation is for the full nonlinear system at $1: 1$ resonance but with non-Boussinesq fluids of densities $\rho_{1}=700 \mathrm{~kg} / \mathrm{m}^{3}$ and $\rho_{2}=1000 \mathrm{~kg} / \mathrm{m}^{3}$. Take the fluids to be quiescent (6.4). Take the initial condition for the interface wave profile at $t=0 \mathrm{sec}$ as in (6.5) with the input 


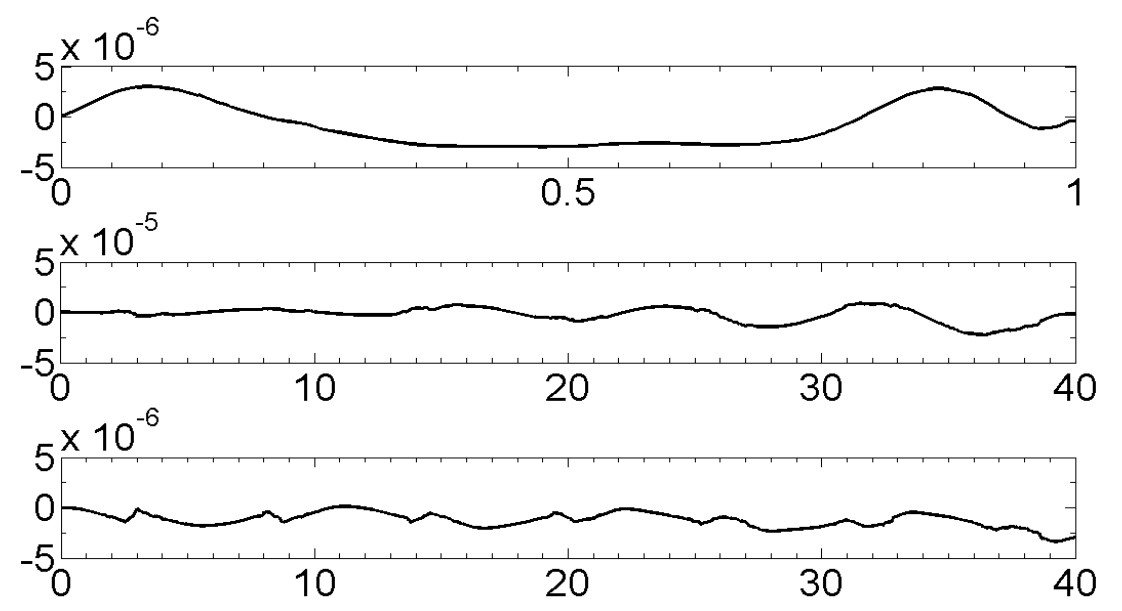

Figure 28: First row: $h_{1} u_{1}+h_{2} u_{2}$ at $t=40 \mathrm{sec}$. Second row: $h_{1}+h_{2}-d$ at $x=.50 \mathrm{~m}$ versus time. Third row: $h_{1} u_{1}+h_{2} u_{2}$ at $x=.50 \mathrm{~m}$ versus time.

parameters

$$
\begin{aligned}
\widehat{q} & =.1 \mathrm{~m}, \quad L=1 \mathrm{~m}, \quad \nu_{1}=\nu_{1: 1}=189.40 \mathrm{~kg} / \mathrm{sec}^{2}, \quad \nu_{2}=-\nu_{1: 1}=-189.40 \mathrm{~kg} /\left(\mathrm{sec}^{2} \mathrm{~m}^{2}\right), \\
h_{1}^{0} & =.04 \mathrm{~m}, \quad h_{2}^{0}=.04 \mathrm{~m}, \quad G=.03 \mathrm{~m} / \mathrm{sec}, \quad \omega_{1}^{\text {resonance }}=1.6534 \mathrm{rad} / \mathrm{sec}, \\
\Delta x & =.01 \mathrm{~m}, \quad \Delta t=.001 \mathrm{sec}, \quad g=9.81 \mathrm{~m} / \mathrm{sec}^{2}, \quad m_{v}=.05\left(\mathrm{~m}_{f}^{1}+\mathrm{m}_{f}^{2}\right)=3.4 \mathrm{~kg}, \\
m_{f}^{1} & =28 \mathrm{~kg}, \quad m_{f}^{2}=40 \mathrm{~kg}, \quad \mathrm{R}=32.71, \quad \mathrm{G}=322.90, \quad \omega_{1}^{f}=.8267 \mathrm{rad} / \mathrm{sec}, \\
\omega_{f v} & =1.6287 \mathrm{rad} / \mathrm{sec}, \quad T^{\text {coupled }}=3.80 \mathrm{sec}, \quad s_{2}=\pi .
\end{aligned}
$$

And take the initial conditions for the vessel position and velocity at $t=0 \mathrm{sec}$ as in (6.5) but with $\widehat{q}=.01 \mathrm{~m}$. The snapshots of the computed nonlinear interface wave profile at $1: 1$ resonance are shown in Figure 29 up to $30 \mathrm{sec}$. The vessel displacement for the full nonlinear coupled system is plotted in Figure 30. The first row in Figure 30 shows the computed numerical solution versus time, the second row shows the energy of the fluid (6.2) versus time for the nonlinear system, and the third row shows the energy of the vessel (6.3) versus time up to $30 \mathrm{sec}$. The partition of energy between the fluid and vessel motion is maintained. The system constraints are preserved approximately with an error of order $\Delta t^{p}$ with $1<p<2$.

\section{Concluding remarks}

The paper is devoted to the formulation of the dynamic coupling between the two-layer inviscid, incompressible and immiscible shallow-water sloshing and the horizontal vessel motion subject to the rigid-lid constraint. The nonlinear coupled system is linearised and a characteristic equation is derived for the natural frequencies of the coupled fluid-vessel oscillations. It is shown that there is an internal $1: 1$ resonance in shallow water limit where symmetric fluid modes are coupled to the vessel motion. The high-resolution f-wave-propagation finite volume methods are adapted to include the exact expression for the pressure gradient at the rigid-lid in the numerical calculations. This is done by considering the pressure gradient as a source term 

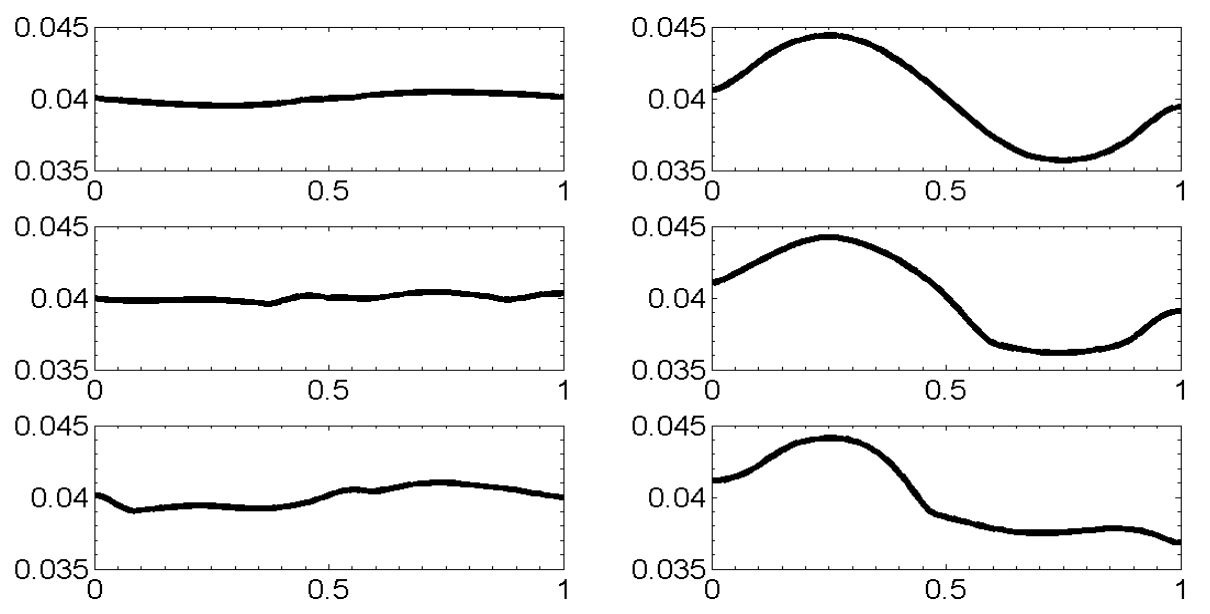

Figure 29: (colour online) Snapshots of the nonlinear interface wave profile for two nonBoussinesq fluids of densities $\rho_{1}=700 \mathrm{~kg} / \mathrm{m}^{3}$ and $\rho_{2}=1000 \mathrm{~kg} / \mathrm{m}^{3}$ at $t=1.9 \mathrm{~s}, t=7.6 \mathrm{~s}$ for the first row from left to right, $t=17.1 \mathrm{~s}, t=22.8 \mathrm{~s}$ for the second row from left to right, and $t=28.5 s, t=30 s$ for the third row from left to right.
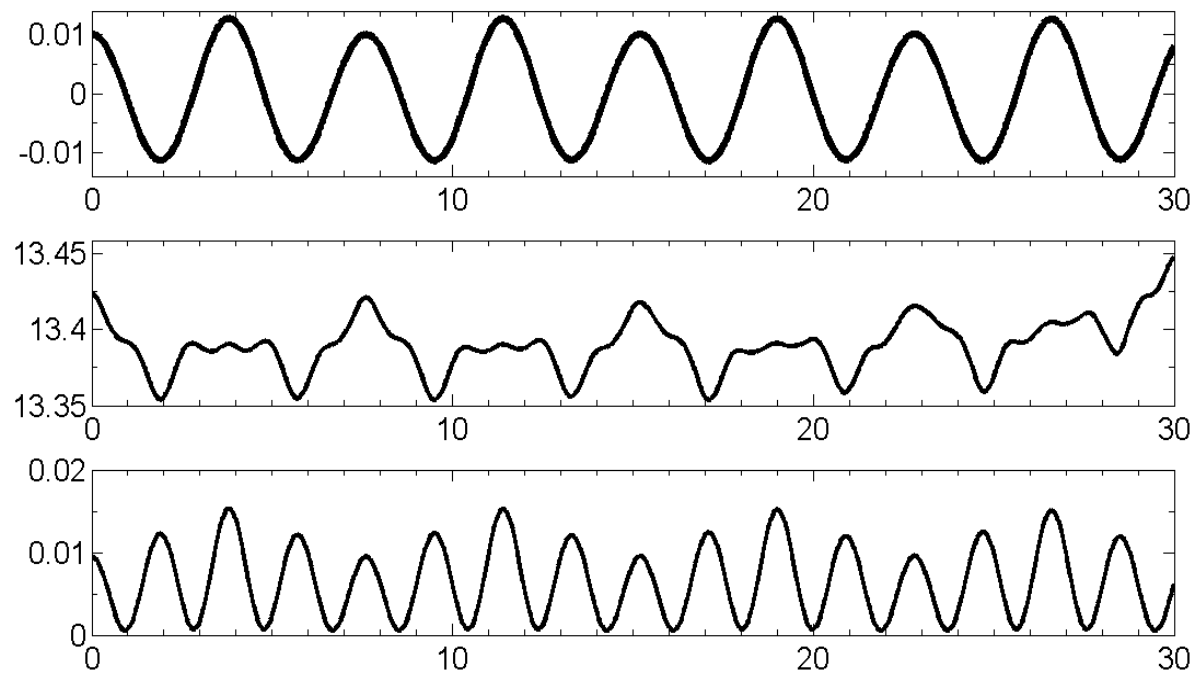

Figure 30: (colour online) First row: computed numerical solution for the vessel motion for the full nonlinear coupled system at $1: 1$ resonance and with two non-Boussinesq fluids. The vertical axis is $q(m)$ and the horizontal axis is time. Second row: nonlinear fluid energy versus time. Third row: vessel energy versus time.

which is included in the jump in the flux vectors before decomposing the differences into waves propagating out from each grid cell interface. The pressure gradient is related to the vessel acceleration which is explicitly time-dependent. The time dependent source terms are handled via a fractional step approach. The MATLAB ODE45 built in explicit Runge-Kutta solver is used to integrate the vessel equation in time.

A direction of great interest is to modify the coupled nonlinear system (1.1) to include the bottom topography and variable cross-section of the vessel into the fluid equations, and study the 
coupled dynamics with influx-efflux boundary conditions at the two ends of the vessel, motivated by the dynamic coupling problem in the ocean wave energy converter (WEC) proposed by Offshore Wave Energy Ltd (OWEL), a schematic of which can be found on the website [1]. OWEL is a floating rectangular device, open at one end to allow waves in. Once they are trapped, the waves undergo interior fluid sloshing. A rise in the wave height is induced within the duct. The wave then creates a seal with the rigid lid resulting in a moving trapped pocket of air ahead of the wave front which drives the power take off.

So the strategy going forward is to modify the current dynamic coupling solver to include a more complicated source term, due to the bottom topography and variable cross-section of the vessel, into the numerical calculations and study how best the time-dependent source terms can be handled. See [7, 4] for a numerical study of the two-layer shallow-water equations in a stationary container subject to the rigid-lid constraint and with open boundary conditions.

In the case of dynamic coupling with two interior fluids of large density difference, like the problem of two-fluid flows inside the OWEL WEC, the current approach in approximating the eigenvalues of the two fluid system with the fractional step method for handling the timedependent source terms may lead to a linear growth of error in the preservation of the system constraints. While this error is tolerable, the study of how the computational strategy could be modified for the numerical simulations is an interesting topic for further study.

\section{Acknowledgments}

The research reported in this paper is supported by the Engineering and Physical Sciences Research Council Grant EP/K008188/1. Due to confidentiality agreements with research collaborators, supporting data can only be made available to bona fide researchers subject to a non-disclosure agreement. Details of the data and how to request access are available from the University of Surrey publications repository: researchdata@ surrey.ac.uk

\section{References}

[1] http://www.owel.co.uk/

[2] R. AbGRALl \& S. KARni. Two-layer shallow water systems: a relaxation approach, SIAM J. Sci. Comput. 31 1603-1627 (2009).

[3] H. Alemi Ardakani. Rigid-Body Motion with Interior Shallow-Water Sloshing, Ph.D. Thesis, University of Surrey, UK (2010).

[4] H. Alemi Ardakani. Theory and application of $f$-wave finite volume methods to the two-layer shallow-water equations with a rigid-lid, Internal Report, University of Surrey (2014).

[5] H. Alemi ARdakAni \& T.J. BRIDGes. Dynamic coupling between shallow-water sloshing and horizontal vehicle motion, Euro. Jnl of Applied Mathematics 21 479-517 (2010). 
[6] H. Alemi ArdakAni, T.J. Bridges \& M.R. TuRner. Resonance in a model for Cooker's sloshing experiment, Euro. J. Mech. B/Fluids 36 25-38 (2012).

[7] H. Alemi Ardakani, T.J. Bridges \& M.R. Turner. Adaptation of f-wave finite volume methods to the two-layer shallow-water equations in a moving vessel with a rigid-lid, Preprint (2014).

[8] P.G. BAIneS. Topographic Effects in Stratified Flows, Cambridge University Press (1995).

[9] D. Bale, R.J. LeVeque, S. Mitran \& J.A. Rossmanith. A wave propagation method for conservation laws and balance laws with spatially varying flux functions, SIAM J. Sci. Comput. 24 955-978 (2002).

[10] A. BoOnKaSAme \& P. MilewsKi. The stability of large-amplitude shallow interfacial non-Boussinesq flows, Studies in Applied Mathematics 128 40-58 (2011).

[11] F. Bouchut \& T. Morales DE LunA. An entropy satisfying scheme for two-layer shallow water equations with uncoupled treatment, ESAIM: M2AN 42 683- 698 (2008).

[12] T.J. BRIDGES \& N.M. Donaldson. Reappraisal of criticality for two-layer flows and its role in the generation of internal solitary waves, Phys. Fluids 19072111 (2007).

[13] I. CAGLAYAN \& R.L. StORCH. Stability of fishing vessels with water on deck: a review, J. Ship Research 26 106-116 (1982).

[14] M.J. Castro-Díaz, E.D. Fernández-Nieto, J.M. González-Vida \& C. ParésMADROÑAL. Numerical treatment of the loss of hyperbolicity of the two-layer shallowwater system, J Sci Comput 48 16-40 (2011).

[15] M. Castro, J. Macías \& C. PARÉs. A Q-scheme for a class of systems of coupled conservation laws with source term. Application to a two-layer 1-D shallow water system, Esaim-Mathematical Modelling and Numerical Analysis-Modelisation Mathematique Et Analyse Numerique 35 107-127 (2001).

[16] M.J. COOKER. Water waves in a suspended container, Wave Motion 20, 385-395 (1994).

[17] B. Di Martino, P. Orenga, \& M. Peybernes. On a bi-layer shallow water model with rigid-lid hypothesis, Math. Mod. Meth. Appl. Sci. 15 843-869 (2005).

[18] F. Dubois, N. Petit \& P. Rouchon. Motion planning and nonlinear simulations for a tank containing fluid, European Control Conference, Karlsruhe, 6 pages (1999).

[19] O.M. Faltinsen \& A.N. Timokha. Sloshing, Cambridge University Press: Cambridge (2009).

[20] J.T. Feddema, C.R. Dohrmann, G.G. Parker, R.D. RobinetT, V.J. Romero \& D.J. SCHMITT. Control for slosh-free motion of an open container, IEEE Control Systems Magazine 17 29-36 (1997).

[21] D.L. GeORGE. Finite Volume Methods and Adaptive Refinement for Tsunami Propagation and Inundation, Ph.D. Thesis, University of Washington, Seattle, WA (2006). 
[22] D.L. GEORGE. Augmented Riemann solvers for the shallow water equations over variable topography with steady states and inundation, Journal of Computational Physics 227 30893113 (2008).

[23] M. GRundelius \& B. Bernhardsson. Control of liquid slosh in an industrial packaging machine, IEEE Int. Conf. Control Appl., Kohala Coast, Hawaii, 6 pages (1999).

[24] A. HERCZYŃSKi \& P.D. WEIDMAN. Experiments on the periodic oscillation of free containers driven by liquid sloshing, J. Fluid Mech. 693 216-242 (2012).

[25] R.A. IbRahim. Liquid Sloshing Dynamics, Cambridge University Press (2005).

[26] Q. JiAng \& R.B. SMith. Ideal shocks in a 2-layer flow. Part I: under a rigid lid, Tellus 53 129-145 (2001).

[27] J. KIM. Finite Volume Methods for Tsunami Generated by Submarine Landslides, Ph.D. Thesis, University of Washington, Seattle, WA (2014).

[28] J. KIM \& R.J. LEVEQUE. Two-layer shallow water system and its applications, Proceedings of 12th International Conference on Hyperbolic Problems, College Park, Maryland (2008).

[29] G.A. LAWRENCE. On the hydraulics of Boussinesq and non-Boussinesq two-layer flows, J. Fluid Mech. 215 457-480 (1990).

[30] R.J. LEVEQUE. Wave propagation algorithms for multidimensional hyperbolic systems, Journal of Computational Physics 131 327-353 (1997).

[31] R.J. LeVeque. Finite Volume Methods for Hyperbolic Problems, Cambridge Texts in Applied Mathematics, Cambridge University Press, Cambridge, UK (2002).

[32] R.J. LeVeque \& D.L. George. High-Resolution Finite Volume Methods for the Shallow Water Equations with Bathymetry and Dry States, In Advanced Numerical Models for Simulating Tsunami Waves and Runup, P. L-F. Liu, H. Yeh \& C. Synolakis, editors, Advances in Coastal and Ocean Engineering, World Scientific 10 43-73 (2007).

[33] D. MACKEY \& E.A. CoX. Dynamics of a two-layer fluid sloshing problem, IMA J. Appl. Math. 68 665-686 (2003).

[34] K.T. MAndLI. Finite Volume Methods for the Multilayer Shallow Water Equations with Applications to Storm Surge, Ph.D. Thesis, University of Washington, Seattle, WA (2011).

[35] K.T. MANDLI. A numerical method for the two layer shallow water equations with dry states, Ocean Modelling 72 80-91 (2013).

[36] N.N. Moiseyev \& V.V. Rumyantsev. Dynamic Stability of Bodies Containing Fluid, Springer (1968).

[37] L.V. OvsyanniKov. Two-layer shallow water model, Journal of Applied Mechanics and Technical Physics 20 127-135 (1979).

[38] C. PRIEuR \& J. DE HalleuX. Stabilization of a 1-D tank containing a fluid modeled by the shallow water equations, Systems \& Control Letters 52, 167-178 (2004). 
[39] M. LA RoccA, G. Sciortino, \& M.A. Boniforti. Interfacial gravity waves in a twofluid system, Fluid Dyn. Res. 30 31-66 (2002).

[40] M. La Rocca, G. Sciortino, C. Adduce, \& M.A. Boniforti. Experimental and theoretical investigation on the sloshing of a two-liquid system with a free surface, Phys. Fluids 17062101 (2005).

[41] J.B. SCHIJF \& J.C. SCHÖNFLED. Theoretical considerations on the motion of salt and fresh water, Proc. of Minn. Int. Hydraulics Conv., Joint meeting IAHR and Hyd. Div ASCE., IAHR 321-333 (1953).

[42] A.L. STewart \& P.J. Dellar. Multilayer shallow water equations with complete Coriolis force. Part I: Derivation on a non-traditional beta-plane, J. Fluid Mech. 690 16-50 (2012).

[43] M.R. Turner, H. Alemi ArdakAni \& T.J. BRidges. Instability of sloshing motion in a vessel undergoing pivoted oscillations, J. Fluids Struct. 52 166-180 (2015).

[44] M.R. Turner, T.J. Bridges \& H. Alemi Ardakani. Dynamic coupling in Cooker's sloshing experiment with baffles, Phys. Fluids 25(10) 112102 (2013).

[45] M.R. TuRneR \& T.J. BRIDGES. Nonlinear energy transfer between fluid sloshing and vessel motion, J. Fluid Mech. 719 606-636 (2013).

[46] M.P. Tzamtzi \& N.D. KouvaKas. Sloshing control in tilting phases of the pouring process, Inter. J. Math., Phys. Eng. Sciences 1 175-182 (2007).

[47] M.P. Tzamtzi \& N.D. KouvaKas. Robustness of a robot control scheme for liquid transfer, in Novel Algorithms and Techniques in Telecommunications, Automation and Industrial Electronics, Edited by T. SoвH ET AL., Springer-Verlag 154-161 (2008).

[48] S. AUS DER WIESCHE. Computational slosh dynamics: theory and industrial application, Comp. Mech. 30 374-387 (2003). 